\title{
La description du Grand Temple de Mexico par Bernardino de Sahagún (Codex de Florence, annexe du Livre II)
}

\section{Aurélie Couvreur}

\section{(2) OpenEdition}

\section{Journals}

Édition électronique

URL : https://journals.openedition.org/jsa/2742

DOI : $10.4000 /$ jsa. 2742

ISSN : 1957-7842

Éditeur

Société des américanistes

\section{Édition imprimée}

Date de publication : 1 janvier 2002

Pagination : 9-46

ISSN : 0037-9174

Référence électronique

Aurélie Couvreur, «La description du Grand Temple de Mexico par Bernardino de Sahagún (Codex de Florence, annexe du Livre II) », Journal de la Société des américanistes [En ligne], 88 | 2002, mis en ligne le 05 janvier 2007, consulté le 15 mars 2022. URL : http://journals.openedition.org/jsa/2742 ; DOI : https://doi.org/10.4000/jsa.2742 


\title{
ARTICLES
}

\section{LA DESCRIPTION DU GRAND TEMPLE DE MEXICO PAR BERNARDINO DE SAHAGÚN} (CODEX DE FLORENCE, ANNEXE DU LIVRE II)

\author{
Aurélie COUVREUR *
}

L'annexe du livre il du Codex de Florence, présentant les 78 édifices réunis sous le nom de « Grand Temple » de Mexico, est une source majeure pour la connaissance du centre cérémoniel aztèque aujourd'hui partiellement redécouvert grâce à l'archéologie. Cet article propose une analyse détaillée de ce témoignage de Sahagún, afin de mettre en évidence sa cohérence interne ainsi que le caractère parfois totalement inédit des informations qu'il contient, notamment au sujet de dieux méconnus et de rites mineurs. La confrontation de ces données avec les autres sources permet d'évaluer la validité de ce texte et de souligner les références constantes aux différents quartiers (calpulli) de Mexico. Enfin, l'hypothèse selon laquelle cette liste renvoie à une image d'enceinte - dispositif que Sahagún a déjà pratiqué dans ses Primeros Memoriales - est évaluée, et une tentative de reconstitution de cette image est proposée.

Mots CLÉs : Mexique central, aztèque, religion, Grand Temple, Codex de Florence, calpulli.

La descripción del Templo Mayor por Bernardino de Sahagún (Códice Florentino, anexo del Libro 2)

El anexo del Libro 2 del Códice Florentino, que describe los 78 edificios conocidos bajo el nombre de "Templo Mayor » de México es una fuente capital para el conocimiento del centro ceremonial azteca, ahora parcialmente descubierto de nuevo gracias a la arqueología. En este artículo se propone un análisis detallado del testimonio de Sahagún, para poner de manifiesto su coherencia interna, así como el carácter, a veces, totalmente inédito de las informaciones que contiene, especialmente a propósito de ciertos dioses desconocidos y ritos de menor importancia. La confrontación de estos datos con las otras fuentes permite evaluar la validez de este texto y subrayar las referencias constantes a los diferentes barrios (calpulli) de México. Por fin, se presenta la hipótesis según la cual dicha lista hace referencia a una imagen de recinto, dispositivo

* Aspirant du FNRS (Fonds National de la Recherche Scientifique, Belgique), Université Libre de Bruxelles, 50 av. Franklin D. Roosevelt, CP 175, 1050 Bruxelles, Belgique. Adresse électronique : ACouvreur@ibelgique.com

Journal de la Société des Américanistes, 2002, 88 : p. 9 à 46. Copyright $@$ Société des Américanistes. 
ya utilizado por Sahagún en sus Primeros Memoriales, y se propone una reconstrucción posible de aquella imagen.

Palabras Claves : México central, azteca, religión, Templo Mayor, Códice Florentino, calpulli.

The description of the Great Temple of Mexico by Bernardino de Sahagín (Florentine Codex, Appendix of Book 2)

The appendix of Florentine Codex's Book 2, describing the 78 buildings of the Great Temple of Mexico, is a major source of knowledge of the Aztec ceremonial centre today rediscovered thanks to archaeology. This paper proposes a detailed analysis of Sahagún's testimony in order to underline its internal coherence as well as the sometimes completely new nature of the information it contains, notably concerning unknown gods and minor rituals. The comparison of these data with the other sources enables us to evaluate this text's validity and to emphasize its constant references to the different districts (calpulli) of Mexico-Tenochtitlan. Eventually, the assumption that this list refers to a representation of an enclosure, a system already used in Sahagún's Primeros Memoriales, is tested and a potential reconstitution of it is proposed.

Key woriss : Central Mexico, Aztec, religion, Great Temple, Florentine Codex, calpulli.

La description de l'enceinte cérémonielle de Mexico contenue dans l'annexe du livre II du Codex de Florence ${ }^{1}$ est une source incontournable pour la connaissance du centre religieux de la capitale aztèque. Elle l'est, non seulement en raison de la richesse exceptionnelle et de la fiabilité de ce codex et des autres écrits de Bernardino de Sahagún, mais aussi et surtout parce qu'elle est une des rares sources présentant de manière détaillée l'ensemble de l'enceinte. En effet, comme le centre de la ville aztèque a été détruit dès la prise de la ville par les Espagnols et qu'il a été très vite reconstruit, des recherches archéologiques extensives ne sont pas envisageables dans cette zone. Les données archéologiques la concernant sont donc fiables, mais partielles. Par ailleurs, les autres descriptions de la ville qui nous sont parvenues brossent un portrait très succinct de l'enceinte cérémonielle, appelée « Grand Temple » ${ }^{2}$ par les Espagnols.

Si ces onze folios de description sont incontournables, ils ne sont pas pour autant simples à interpréter. En effet, ce que les informateurs de Sahagún présentent comme la description de "tous les temples des Mexicains " ${ }^{3}$ est, en réalité, une suite de 78 descriptions de lieux variés dont certains aspects sont intrigants.

Un des éléments surprenants est le nombre d'«édifices» censés se trouver à l'intérieur de l'enceinte : 78. En effet, une rapide comparaison avec les autres sources écrites met en évidence l'extravagance de ce chiffre. Il apparaît cependant que toutes les descriptions ne s'accordent pas sur la nature des objets comptabilisés ce qui, nous le verrons, peut, d'une part, expliquer les divergences entre les descriptions de l'époque et, d'autre part, concorder avec la réalité archéologique du site ${ }^{4}$.

Un autre aspect curieux est lié au fait que, contre toute attente, la description des informateurs de Sahagún ne s'attache pas à l'aspect physique et architectural de l'enceinte. Ils ne précisent ainsi jamais la localisation des lieux les uns par rapport aux autres, et les indications concernant l'aspect des édifices sont, d'une part, quelques 
remarques générales introduites dans la première description, celle du temple de Huitzilopochtli, et, d'autre part, des indications distillées par Sahagún dans sa version espagnole, sur l'origine desquelles il conviendra de s'interroger. À défaut de données architecturales, les informateurs du Codex de Florence présentent les 78 lieux selon un schéma assez régulier, en mentionnant le nom de l'édifice et en évoquant ensuite les personnes qui le fréquentaient, les rites qui y étaient pratiqués et les moments de l'année durant lesquels le lieu était utilisé. Il s'agit donc d'une description de l'enceinte selon un point de vue rituel, ce qui, somme toute, est logique pour une annexe du livre II du Codex de Florence... Cependant, le plus étonnant réside sans doute dans les données concernant les rites effectués dans ces lieux. En effet, non seulement les informateurs ne s'attardent pas sur les édifices dédiés aux dieux principaux du panthéon aztèque, mais, de plus, ils affirment qu'un bon nombre d'édifices de l'enceinte étaient consacrés à des dieux mal connus, à propos desquels ils décrivent des rites particulièrement mal documentés. Pour tenter de comprendre ces éléments inattendus, le contenu de chaque description a été étudié ${ }^{5}$, pour essayer de dégager un fil conducteur de la liste et de déterminer dans quelle mesure celle-ci renvoie aux édifices contenus dans l'enceinte de Mexico-Tenochtitlan.

\section{LES DESCRIPTIONS D'AUTRES CHRONIQUEURS}

Plusieurs chroniques contiennent une description générale du centre cérémoniel de Mexico : la seconde lettre de Cortés, l'unique témoin direct à avoir rédigé sa description, le Libro de los ritos de Durán (1984, 1, p. 20) et l'Historia d'Oviedo (1945, 10, p. 53). Motolinía (1995, pp. 50-51), qui aflirme avoir vu la pyramide double de Mexico, ne donne que des informations générales à propos des grands centres cérémoniels. Quant à Torquemada (1969, 2, pp. 144-147), il fournit, d'une part, une savante compilation de données provenant d'autres descriptions, et notamment de celles mentionnées ci-dessus, et, d'autre part, une copie de la liste de Sahagún, annotée de commentaires personnels, très souvent erronés.

Tous les chroniqueurs s'accordent à dire que le centre religieux de Mexico était enfermé dans un mur d'enceinte, souvent comparé à une muraille de forteresse. Oviedo (1945, 10, p. 53) affïrme que l'espace dégagé à l'intérieur des murailles est long de plus d'un tir d'arbalète, soit environ 400 mètres. D'après Durán (1984, 1, p. 22), cette enceinte était percée de quatre ouvertures domnant sur les chaussées qui quittaient Tenochtitlan. La pyramide soutenant les temples de Tlaloc et de Huitzilopochtli est unanimement décrite comme l'édifice le plus grand de l'enceinte. Tous les chroniqueurs indiquent qu'elle était entourée d'autres édifices : des temples d'autres dieux, de taille moins imposante, des résidences de prêtres et encore d'autres pièces dont l'usage n'est pas toujours spécifié. Torquemada (1969, 2, p. 146) ajoute que chaque entrée était associée à une grande pièce servant de « salle d'armes ».

Le nombre de temples et d'édifices annexes cité dans la description générale de l'enceinte varie fort d'un chroniqueur à l'autre (Figure 1). En réalité, la variété de ces chiffres correspond au moins en partie aux types d'édifices comptabilisés par les chroniqueurs qui, rappelons-le, travaillent à partir d'informations indirectes.

Les deux auteurs qui donnent des chiffres bas, Durán et Motolinía, précisent que les temples étaient tous entourés de plusieurs salles, dont les usages étaient multiples. 
Il apparaît donc que le chiffre de huit à quinze édifices correspond aux temples sur pyramide, ce qui est d'ailleurs clairement précisé par Motolinía. Au fil de sa description des rites, Durán mentionne et décrit dix temples qui correspondent aux divinités principales du panthéon aztèque, à l'exception du temple de Xiuhtecuhtli, dont l'absence est étonnante (Figure 2a).

\begin{tabular}{|l|l|l|}
\hline Chroniqueur & Nombre & Type d'édifice \\
\hline Cortés $(1996,130)$ & 40 & Tours $($ torres $)$ \\
\hline Durán $(1984,120)$ & 8 ou $9^{*}$ & Temples $($ templos $)$ \\
\hline Motolinía $(1995,51)$ & 12 ou 15 & Teocalli \\
\hline Oviedo $(1945,53)$ & 60 & Cus $($ Qües $)$ \\
\hline Torquemada $(1969,145)$ & Plus de 40 & $\begin{array}{l}\text { Temples et tours plus petites }(\text { tem- } \\
\text { plos, } \text {, torres menores })\end{array}$ \\
\hline
\end{tabular}

*Au fil de son Libro de los ritos, Durán mentionne dix temples, qui correspondent aux temples des divinités principales.

FIG. 1. - Nombre et types des structures comptabilisées à l'intérieur de l'enceinte de Mexico, d'après différentes chroniques.

\begin{tabular}{|c|c|}
\hline a. Durán & b. Liste du Codex de Florence \\
\hline $\begin{array}{l}\text { 1. Grand Temple (temple de Huitzilopochtli } \\
\text { et de Tlaloc) }\end{array}$ & $\begin{array}{l}\text { Ensemble 1: temple de Huitzlilopochtli et } \\
\text { Tlaloc }\end{array}$ \\
\hline 2. Temple de Tezcatlipoca & Ensemble 6 : quauhxicalco de Tezcatlipoca \\
\hline $\begin{array}{l}\text { 3. Temple de Cihuacoatl (la chambre est } \\
\text { appelée Tlillan) }\end{array}$ & Ensemble 3 : temple Tlillan \\
\hline 4. Temple de Chicomecoatl & Ensemble 15: Cinteupan \\
\hline 5. Temple de Mixcoatl (Mixcoateocalli) & Ensemble 2 : temple de Mixcoatl \\
\hline 6. Temple du soleil & Ensemble 4 : Huey Quauhxicalco $\left(\mathrm{n}^{\circ} 8\right)$ \\
\hline 7. Temple de Quetzalcoatl & $\begin{array}{l}\text { Ensemble } 18: \text { temple de Quetzalcoatl- } \\
\text { «9 vent }(?)\end{array}$ \\
\hline $\begin{array}{l}\text { 8. Temple de tous les " saints » (temple de } \\
\text { Mexico où l'image de Toci était gardée) }\end{array}$ & $\begin{array}{l}\text { Ensemble } 23 \text { ? : édifices dédiés aux Cihuate- } \\
\text { teo }\end{array}$ \\
\hline 9. Temple de Xipe & Ensemble 21 : Yopico \\
\hline 10. Temple de Xochiquetzal & $\begin{array}{l}\text { Ensemble } 22 ? \text { : temple de Macuilmalinalli, } \\
\text { utilisé en Xochihuitl qui, selon Durán, est le } \\
\text { jour de la fète de Xochiquetzal. }\end{array}$ \\
\hline
\end{tabular}

Fıg. 2 a. - Temples cités dans le Libro de los ritos de Durán.

b. - Identification des temples cités par Durán au sein de la liste du Codex de Florence, sur la base des complexes identifiés dans la Figure 4.

Cortés (1996, pp. 129-131), lui, aftirme que les « tours », qui sont sans doute des temples sur pyramide, étaient au nombre de 40 , chiffre auquel il faut donc ajouter les salles servant à la résidence des prêtres qu'il mentionne également. Le conquistador, 
seul témoin direct, aurait donc compté au moins deux fois plus de structures hautes que les autres chroniqueurs. Cette différence de proportion doit toutefois être tempérée par la tendance à l'exagération ${ }^{6}$ du futur Marqués del Valle, qui s'exprime sans doute encore ici. Quant au chiffre de Torquemada, il est difficilement utilisable, tant il paraît évident que sa description tardive n'est en fait qu'une composition à partir de différentes sources juxtaposées. En l'occurrence, il reprend ici l'indication de Cortés.

En interprétant textuellement les informations rapportées par Oviedo, il apparaîtrait que pas moins de 60 temples sur pyramide seraient bâtis dans l'enceinte, puisqu'il dénombre autant de cus et que c'est précisément ce terme qu'il utilise pour qualifier la pyramide double. Cependant, il faut souligner le fait que le terme maya $\mathrm{Cu}^{7}$ a pu être employé pour désigner différents types d'édifices, que ce soit des petites structures ou des bâtiments de plus grande taille, comme en témoigne l'usage de ce terme par Sahagún ${ }^{8}$. Le terme $c u$ s'applique donc aux temples sur pyramide, mais également à des structures de taille variable, ayant peut-être comme caractéristique commune le fait d'être accessibles par des marches. Il est dès lors très probable que les $60 \mathrm{cus}$ d'Oviedo se rapportent, eux aussi, à des structures de tailles différentes.

On peut conclure de ces témoignages écrits que l'enceinte comptait probablement une vingtaine de temples sur pyramide, entourés d'un certain nombre d'édifices annexes. Le nombre de ces structures basses ne peut être déterminé avec précision, mais les chiffres qu'avancent Oviedo et Sahagún sont tout à fait vraisemblables. En effet, les recherches archéologiques menées jusqu'à ce jour dans les alentours immédiats de la pyramide double, mais également dans la zone archéologique qui correspond au centre religieux aztèque, indiquent clairement que les édifices y étaient nombreux et fort rapprochés, et que leurs dimensions et leur structure étaient très variées (Barrera Rivera 1999, p. 31).

\section{LA DESCRIPTION DU CODEX DE FLORENCE}

La liste de Sahagún est à la fois plus précise et moins lisible que les autres descriptions. Plus précise, en ce sens qu'elle mentionne l'identité des dieux auxquels les édifices sont dédiés, alors que les autres descriptions ne se concentrent que sur la pyramide double et les temples de Huitzilopochtli et de Tlaloc à son sommet. Mais, par de nombreux aspects, la liste est plus vague, notamment quant au nombre de temples sur pyramide qu'elle évoque. Ce nombre pourrait être déduit par un recensement de tous les iteupan décrits (Figure 3). Cependant, il apparaît clairement que, selon ce critère, une partie des temples sur pyramide seraient dédiés à des dieux presque inconnus, tandis que certains édifices de la liste, qui ne sont pas qualifiés de iteupan, sont connus par ailleurs comme étant des temples sur pyramide. C'est par exemple le cas du Huey quauhxicalco $\left(n^{\circ} 8\right)$, qui est très probablement le temple du soleil décrit par Durán (1984, 1, p. 106).

On peut également tenter d'identifier les édifices en tenant compte des qualificatifs qui les désignent, car certains d'entre eux ne se rapportent qu'à des édifices bas. Cependant, cette méthode ne permet de discerner que trente structures basses :

- Six édifices sont des tzompantli ou plates-formes d'exposition des crânes ${ }^{9}$; 


\begin{tabular}{|c|c|c|c|c|c|c|c|c|c|c|c|c|c|c|c|c|}
\hline & Lieu & 节 & 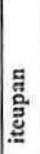 & $\begin{array}{l}\text { 异 } \\
\text { E⿱ } \\
\text { है }\end{array}$ & 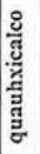 & 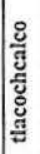 & 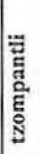 & 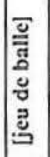 & 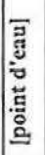 & 产 & z & 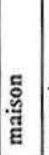 & तूّ̆ & 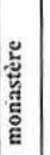 & हूँ & $\begin{array}{l}\text { Description de } \\
\text { Sahagún }\end{array}$ \\
\hline 1 & $\begin{array}{l}\text { Temple de } \\
\text { Huitzilopochtli }\end{array}$ & $x$ & & & & & & & & $\mathrm{x}$ & & & & & & Temple \\
\hline 2 & $\begin{array}{l}\text { Tlalocan, temple } \\
\text { d'Epcoatl }\end{array}$ & & $x$ & & & & & & & & $x$ & & & & & $c u$ principal \\
\hline 3 & $\begin{array}{l}\text { Macuilcalli ; } \\
\text { Macuilquiauitl }\end{array}$ & & & & & & & & & & $\mathrm{x}$ & & & & & $c u$ \\
\hline 4 & Teccizcalli & & & & & & & & & & & $x$ & & & & maison \\
\hline 5 & Poyauhtlan & & & & & & & & & & & & & & & I \\
\hline 6 & Tzompantli de Mixcoatl & & & & & & $x$ & & & & & & & & $\mathrm{x}$ & $\begin{array}{l}\text { édifice dans lequel ils } \\
\text { embrochaient les têtes }\end{array}$ \\
\hline 7 & Tlalxicco & & & & & & & & & & $x$ & & & & & $c u$ \\
\hline 8 & Huey Quauhxicalco & & & & $x$ & & & & & & & & $x$ & & & oratoire \\
\hline 9 & Tochinco & & & & & & & & & & $x$ & & & & & $\begin{array}{l}\text { cu bas, carré, avec des } \\
\text { marches sur les } 4 \\
\text { faces }\end{array}$ \\
\hline 10 & Teotlalpan & & & & & & & & & & & & & & $\mathrm{x}$ & $\begin{array}{l}\text { bosquet entouré de } 4 \\
\text { parois }\end{array}$ \\
\hline II & Tlilapan & & & & & & & & $\mathrm{x}$ & & & & & & $\mathrm{x}$ & $\begin{array}{l}\text { source comme un } \\
\text { bassin }\end{array}$ \\
\hline 12 & Tlillan calmecac & & & $x$ & & & & & & & & & $x$ & & & oratoire \\
\hline 13 & Calmecac de Mexico & & & $x$ & & & & & & & & & & $x$ & & monastère \\
\hline 14 & Coacalco & & & & & & & & & & & & & & $x$ & $\begin{array}{l}\text { salle grillagée comme } \\
\text { une prison }\end{array}$ \\
\hline 15 & Quauhxicalco & & & & $x$ & & & & & & $x$ & & & & & $\begin{array}{l}\text { petit } c u \text {, rond, de } 3,5 \\
\text { brasses de large, sans } \\
\text { couverture }\end{array}$ \\
\hline 16 & Autre Quauhxicalco & & & & $x$ & & & & & & $x$ & & & & & $c u$ ou momoztli \\
\hline 17 & Teccalco & & & & & & & & & & $x$ & & & & & $c u$ \\
\hline 18 & Tzompantli & & & & & & $\mathrm{x}$ & & & & & & & & & 1 \\
\hline 19 & Temple de Huitznauac & & $x$ & & & & & & & & $x$ & & & & & $c u$ \\
\hline 20 & Tezcacalco & & & & & & & & & & & & $x$ & & & oratoire \\
\hline 21 & $\begin{array}{l}\text { Tlacochcalco Acatl } \\
\text { Yiacapan }\end{array}$ & & & & & $x$ & & & & & & $x$ & & & & maison \\
\hline
\end{tabular}




\begin{tabular}{|c|c|c|c|c|c|c|c|c|c|c|c|c|c|c|c|c|}
\hline & Lieu & 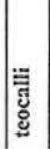 & 恐 & \begin{tabular}{|l} 
ङू \\
छू \\
हु
\end{tabular} & 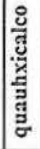 & 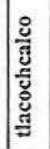 & 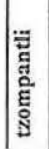 & 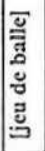 & 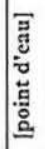 & हूँ & : & 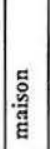 & | & 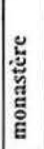 & 苂 & $\begin{array}{l}\text { Description de } \\
\text { Sahagún }\end{array}$ \\
\hline 22 & Teccizcalco & & & & & & & & & & & & $x$ & & & oratoire \\
\hline 23 & Huitztepehualco & & & & & & & & & & & & & & $\mathrm{x}$ & $\begin{array}{l}\text { enclos ou entouré de } \\
4 \text { parois }\end{array}$ \\
\hline 24 & $\begin{array}{l}\text { Calmecac de } \\
\text { Huitznahuac }\end{array}$ & & & $\mathrm{x}$ & & & & & & & & & & $\mathrm{x}$ & & monastère \\
\hline 25 & Quauhxicalco séparé & & & & $x$ & & & & & & $\mathrm{x}$ & & & & & $c u$ \\
\hline 26 & $\begin{array}{l}\text { Temple de } \\
\text { Macuilcipactli }\end{array}$ & & $\mathrm{x}$ & & & & & & & & $\mathrm{x}$ & & & & & grand $c u$ \\
\hline 27 & Calmecac de Tetlanman & & & $x$ & & & & & & & & & & $x$ & & monastère \\
\hline 28 & Temple de Iztac cinteotl & & $\mathrm{x}$ & & & & & & & & $x$ & & & & & $c u$ \\
\hline 29 & Tetlanman & & & & & & & & & & $x$ & & & & & $c u$ \\
\hline 30 & Temple de Chicomecatl & & $x$ & & & & & & & & $\mathrm{x}$ & & & & & $c u$ \\
\hline 31 & Tezcaapan & & & & & & & & $\mathrm{x}$ & & & & & & $\mathrm{x}$ & $\begin{array}{l}\text { source comme un } \\
\text { bassin }\end{array}$ \\
\hline 32 & Tezcatlachco & & & & & & & & & & & & & & $x$ & jeu de "pelote" \\
\hline 33 & Tzompantli & & & & & & $x$ & & & & & & & & & 1 \\
\hline 34 & Tlamatzinco & & & & & & & & & & $\mathrm{x}$ & & & & & $c u$ \\
\hline 35 & Tlamatzinco calmecac & & & $x$ & & & & & & & & & & $x$ & & monastère \\
\hline 36 & Quauhxicalco & & & & $\mathrm{x}$ & & & & & & $\mathrm{x}$ & & & & & $\begin{array}{l}\text { petit et large } \mathrm{cl} \text {, et un } \\
\text { peu concave et } \\
\text { profond }\end{array}$ \\
\hline 37 & Mixcoateupan & & $x$ & & & & & & & & $\mathrm{x}$ & & & & & $c u$ \\
\hline 38 & Netlatiloyan & & & & & & & & & & $\mathrm{x}$ & & & & & $\begin{array}{l}\text { cu au pied duquel se } \\
\text { trouvait une grotte } \\
\text { (cave) }\end{array}$ \\
\hline 39 & Teotlachico & & & & & & & $\mathrm{x}$ & & & & & & & $x$ & jeu de "pelote" \\
\hline 40 & Ilhuicatitlan & & & & & & & & & & & & & & $\mathrm{x}$ & $\begin{array}{l}\text { colonne grosse et } \\
\text { haute }\end{array}$ \\
\hline 41 & Grand tzompantli & & & & & & $x$ & & & & & & & & & I \\
\hline 42 & Mecatlan & & & & & & & & & & & $x$ & & & & maison \\
\hline 43 & Temple du maĩs & & $x$ & & & & & & & & $x$ & & & & & $\mathrm{cu}$ \\
\hline 44 & Temple des Centzontotochtin & & $x$ & & & & & & & & $\mathrm{x}$ & & & & & $c u$ \\
\hline
\end{tabular}




\begin{tabular}{|c|c|c|c|c|c|c|c|c|c|c|c|c|c|c|c|c|}
\hline & Lieu & हีँ & 品 & 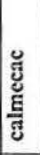 & 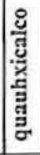 & 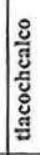 & 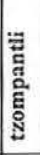 & 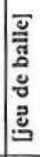 & 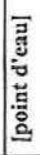 & 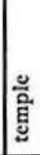 & ปี & 哭 & 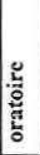 & 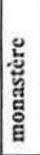 & 氖 & $\begin{array}{l}\text { Description de } \\
\text { Sahagún }\end{array}$ \\
\hline 45 & Temple du maĩs & & $x$ & & & & & & & & $x$ & & & & & $c u$ \\
\hline 46 & Netotiloyan & & & & & & & & & & & & & & $x$ & $\begin{array}{l}\text { lieu ou partie d'un } \\
\text { patio }\end{array}$ \\
\hline 47 & Chililico & & & & & & & & & & $x$ & & & & & $c u$ \\
\hline 48 & Coaapan & & & & & & & & $\mathrm{x}$ & & & & & & $x$ & source \\
\hline 49 & Pochtlan & & & & & & & & & & & & & $x$ & & monastère \\
\hline 50 & Atlauhco & & & & & & & & & & & & & $x$ & & monastère \\
\hline 51 & Yopico & & & & & & & & & & $x$ & & & & & $c u$ \\
\hline 52 & Temple de Yacatecuhtli & & $x$ & & & & & & & & $x$ & & & & & $c u$ \\
\hline 53 & $\begin{array}{l}\text { Temple de } \\
\text { Huitzilinquatec }\end{array}$ & & $x$ & & & & & & & & $x$ & & & & & $c u$ \\
\hline 54 & Calmecac de Yopico & & & $x$ & & & & & & & & & $x$ & $x$ & & monastère ou oratoire \\
\hline 55 & Tzompantli de Yopico & & & & & & $x$ & & & & & & & & & 1 \\
\hline 56 & Tzompantli & & & & & & $x$ & & & & & & & & & 1 \\
\hline 57 & $\begin{array}{l}\text { Temple de } \\
\text { Macuilmalinalli }\end{array}$ & & $x$ & & & & & & & & $x$ & & & & & $c u$ \\
\hline 58 & Aticpac & & & & & & & & & & & & $x$ & & & oratoire \\
\hline 59 & Netlatiloyan & & & & & & & & & & & & & & $\mathrm{x}$ & grotte ou cave \\
\hline 60 & Atlauhco & & & & & & & & & & & & $x$ & & & oratoire \\
\hline 61 & Tzonmolco calmecac & & & $x$ & & & & & & & & & & $x$ & & monastère \\
\hline 62 & Temalacatl & & & & & & & & & & & & & & $\mathrm{x}$ & $\begin{array}{l}\text { pierre comme une } \\
\text { meule de moulin }\end{array}$ \\
\hline 63 & $\begin{array}{l}\text { Temple de } \\
\text { Nappatecuhtli }\end{array}$ & & $x$ & & & & & & & & $x$ & & & & & $c u$ \\
\hline 64 & Tzonmolco & & & & & & & & & & $x$ & & & & & $\mathrm{cu}$ \\
\hline 65 & Coatlan & & & & & & & & & & $x$ & & & & & $\begin{array}{l}c u \text { appelé Coatlan, qui } \\
\text { se trouvait près de cet } \\
\text { oratoire [Atlauhco] }\end{array}$ \\
\hline 66 & Xochicalco & & & & & & & & & & $x$ & & & & & $c u$ \\
\hline 67 & Yopicalco & & & & & & & & & & & $x$ & & & & maison \\
\hline
\end{tabular}




\begin{tabular}{|c|c|c|c|c|c|c|c|c|c|c|c|c|c|}
\hline & Lieu & 局 & 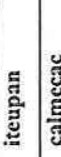 & 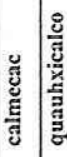 & 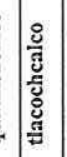 & 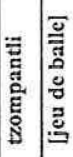 & 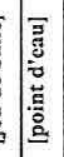 & 䒕 & ป & हू. & 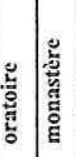 & 苞 & $\begin{array}{l}\text { Description de } \\
\text { Sahagún }\end{array}$ \\
\hline 68 & Toxpalatl & & & & & & $\mathrm{x}$ & & & & & & source \\
\hline 69 & $\begin{array}{l}\text { Tlacochcalco } \\
\text { quauhquiahuac }\end{array}$ & & & & $\mathrm{x}$ & & & & & $x$ & & & maison \\
\hline 70 & Tolnauac & & & & & & & & & $x$ & & & maison \\
\hline 71 & Tilocan & & & & & & & & & $\mathrm{x}$ & & & maison \\
\hline 72 & Itepeioc & & & & & & & & & $\mathrm{x}$ & & & maison \\
\hline 73 & Calpulli de Huitznauac & & & & & & & & & $\mathrm{x}$ & & & maison \\
\hline 74 & Atempan & & & & & & & & & $\mathrm{x}$ & & & maison \\
\hline 75 & Tlacochcalco Tezcacoac & & & & $\mathrm{x}$ & & & & & $x$ & & & maison \\
\hline 76 & $\begin{array}{l}\text { Grand calpulli d'Acatl } \\
\text { Yiacapan }\end{array}$ & & & & & & & & & $\mathrm{x}$ & & & maison \\
\hline 77 & Techielli & & & & & & & & $x$ & & & & petit $c u$ \\
\hline 78 & Calpulli & & & & & & & & & $\mathrm{x}$ & & & $\begin{array}{l}\text { quelques petites } \\
\text { maisons dont était } \\
\text { entouré l'intérieur du } \\
\text { patio lio }\end{array}$ \\
\hline
\end{tabular}

Fig. 3. - Types d'édifices, dans le texte nahuatl (gauche) et la version de Sahagún (droite).

- Sept édifices sont des calmecac, des lieux où résidaient les prêtres et les jeunes gens qui leur étaient confiés. Il faut aussi ajouter le Pochtlan ( $\left.\mathrm{n}^{\circ} 49\right)$ et l'Atlauhco $\left(\mathrm{n}^{\circ} 50\right)$, car leurs descriptions les présentent clairement comme des calmecac, même s'ils ne sont pas désignés comme tels.

- La liste contient deux terrains de jeu de balle ${ }^{10}$ (tlachco) qui, même s'ils étaient de taille importante, peuvent être considérés comme des édifices bas.

- Trois tlacochcalco sont cités et peuvent probablement être identifiés aux «salles d'armes » associées aux entrées de l'enceinte ${ }^{11}$.

- Les quatre points d'eau ou lieux de bains de la liste doivent sans doute être considérés comme des édifices bas ou des petites structures ${ }^{12}$;

- Enfin, une partie des descriptions correspond à des structures qui, sans être à proprement parler des édifices, sont clairement de petite taille : le Huitztepehualco $\left(\mathrm{n}^{\circ} 23\right)$ où étaient dispersées les épines ayant servi à l'autosacrifice; le Netlatiloyan $\left(\mathrm{n}^{\mathrm{o}} 59\right)^{13}$, dont la description indique qu'il s'agit d'une cache cérémonielle; et le Techielli $\left(\mathrm{n}^{\circ} 77\right)$, un lieu de conservation de roseaux axcoyatl, que Sahagún qualifie de petit $\mathrm{cu}$. De même, le lieu aride duquel part la procession pour le Zacatepec en Quecholli, le Teutlalpan ( $\left.{ }^{\circ} 10\right)$, est probablement, comme l'indique Sahagún, un bosquet artificiel entouré d'un muret. 
Restent donc une quarantaine d'édifices à propos desquels on ne peut se prononcer sans avoir recours aux commentaires de Sahagún ou à une autre méthode d'investigation.

L'utilisation des commentaires du franciscain est délicate. En effet, l'essentiel des précisions qu'il ajoute concerne l'aspect physique des édifices. Or il est certain que ce n'est pas sur la base d'une observation directe qu'il connaît ces détails, puisque le Grand Temple était détruit de longue date lorsqu'il composa son Historia. Il est probable que ces détails lui ont été fournis par une image de l'enceinte, une sorte de " plan » illustrant le Grand Temple ${ }^{14}$. Plusieurs indices vont dans ce sens.

Le premier, et non des moindres, est le fait que Sahagún a, au cours de travaux antérieurs, fait établir — ou recueilli — une image d'enceinte accompagnée d'une liste, qui est intégrée dans ses Primeros Memoriales (f. 269 recto) ${ }^{15}$ (Figure 6). Par ailleurs, Torquemada (1969, 2, p. 146) affïrme que Sahagún, à Mexico, a fait peindre une image de l'enceinte cérémonielle et qu'il l'a ensuite envoyée en Espagne. Il ne précise pas si cette image disparue était, elle aussi, associée à une liste, ce qui semble très vraisemblable.

Le second point étayant cette hypothèse est le fait que les commentaires de Sahagún concernent uniquement l'aspect extérieur des édifices et qu'une partie de ces indications s'appliquent mieux à des représentations d'édifices qu'à des constructions réelles. Un des exemples est sa description du Tzompantli $\left(\mathrm{n}^{\circ} 18\right)$, qui est présenté comme «quelques mâts plantés, trois ou quatre, dans lesquels étaient passées quelques hampes, comme des lances », description qui s'applique parfaitement aux images de tzompantli dans les codex, préhispaniques ou non, et notamment à la page des Primeros Memoriales ${ }^{16}$ (Figure 6).

Dans cette perspective, il est possible que les qualificatifs de Sahagún soient déduits d'une représentation de ces édifices. On peut aller plus loin en suggérant que, au sein de cette liste, il aurait qualifié de $c u$ les édifices avec escalier, tandis que les structures qu'il désigne comme « monastère » ou « maison » correspondraient à des édifices bas. Si on applique cette hypothèse, on pourrait décrire l'image de la pierre temalacatl dans les Primeros Memoriales (Figure 6, i) comme un petit $c u$ avec des marches sur les quatre côtés... Malheureusement, comme la liste qui accompagne l'image des Primeros Memoriales n'est pas commentée, il est impossible de tester cette hypothèse.

\section{La structuration de la liste en différents complexes}

L'analyse de la structure de la liste peut donner des indications quant au nombre de temples inscrits dans l'enceinte cérémonielle. En effet, il apparaît clairement que certaines descriptions peuvent être rapprochées les unes des autres et que différents groupes d'édifices peuvent ainsi être dégagés. Ces groupes ont généralement comme point commun leur utilisation lors d'une fête particulière ou la divinité à laquelle ils sont dédiés, ce qui peut indiquer que la liste a été composée, plus ou moins systématiquement, en fonction de ces critères, comme le propose Graulich $\left(1980\right.$, p. 505) ${ }^{17}$. Bien souvent, ces groupes d'édifices peuvent aussi être mis en rapport avec un des calpulli de Tenochtitlan ${ }^{18}$, les "quartiers » de la ville qui correspondaient à des lignages exerçant la même profession et qui rendaient parfois un culte à des divinités tutélaires particulières (Carrasco 1971, pp. 363-366). 
Plusieurs éléments indiquent que les informateurs ont souvent énuméré les différentes parties d'un même ensemble cérémoniel ${ }^{19}$. L'indice le plus évident de la dissociation des annexes est la manière dont les informateurs de Sahagún décrivent la pyramide supportant les temples de Huitzilopochtli et de Tlaloc. En effet, ils évoquent successivement les deux sanctuaires, que Sahagún interprète dès lors comme des édifices différents. Cette distinction est bien sûr défendable au point de vue symbolique, mais elle montre clairement que tous les lieux de la liste ne sont pas des édifices indépendants. À partir de ces constatations, il est possible de regrouper les différentes descriptions et, par là, de recomposer les différents ensembles centrés autour d'une pyramide. Les résultats de cette recomposition sont présentés dans la Figure 4.

\section{Les ensembles d'édifices ${ }^{20}$}

La liste commence avec la présentation du cœur du panthéon aztèque et de l'enceinte cérémonielle : les temples dédiés aux dieux Huitzilopochtli et Tlaloc ( $n^{\circ} 1$ \& 2). Les édifices qui les suivent directement sont également liés à des événements ou des personnes de première importance dans la vie de l'empire. Le MacuilcalliMacuilquiauitl ( $\left.\mathrm{n}^{\circ} 3\right)$, dont le nom signifie « 5 Maison-5 Pluie », ne semble pas particulièrement dédié à ces dieux « $5 »^{21}$, puisqu’il est présenté comme le lieu de sacrifice des espions au service des villes contre lesquelles la Triple Alliance menait la guerre fleurie. Il est possible que ce ou peut-être ces lieux aient fait partie du temple de Huitzilopochtli et de Tlaloc ${ }^{22}$, car c'est à Huitzilopochtli qu'étaient dédiés les sacrifices des guerriers capturés au cours de ces combats (Durán, 2, ch. 28, pp. 232-233). Cette hypothèse, également suggérée par la troisième position de cet édifice au sein de la liste, est renforcée par la découverte récente d'une sculpture d'un dieu « 5 » au nord de la pyramide double de Mexico (Matos Moctezuma 1999, p. 25). Le quatrième édifice, le Teccizcalli, est un lieu de pénitence de Montezuma qui n'est pas associé à un dieu précis. Cependant, comme certains témoignages indiquent que le roi, de retour de campagne militaire victorieuse, se rendait au temple de Huitzilopochtli pour y faire pénitence (Tezozomoc 1943, p. 47), il se peut que le Teccizcalli soit ce lieu de pénitence associé au temple de Huitzilopochtli. Le cinquième lieu est également un lieu de pénitence, celui des prêtres des temples de Huitzilopochtli et de Tlaloc. Ces deux dernières descriptions font donc référence à trois personnages de la plus haute importance : Montezuma II, l'empereur aztèque, et les deux prêtres principaux ${ }^{23}$. À ces premiers lieux, on peut ajouter le Calmecac de Mexico $\left(\mathrm{n}^{\circ} 13\right)$, le lieu de résidence des pénitents ${ }^{24}$ de Tlaloc, et peut-être aussi le Coacalco ( $\left.n^{\circ} 14\right)$, une « prison » pour les images de dieux ennemis capturés ${ }^{25}$. Les informateurs ne précisent pas à quel dieu était dédié le Grand Tzompantli ( $\left.\mathrm{n}^{\circ} 41\right)$, mais l'indication de Sahagún selon laquelle cette plate-forme était associée au temple de Huitzilopochtli est très vraisemblable, puisque les informateurs de Sahagún affĭrment qu’il était utilisé en Panquetzaliztli et qu'on sait par ailleurs qu'un tzompantli joue un rôle majeur au cours de ce mois dédié à Huitzilopochtli ${ }^{26}$. Le Tilocan $\left(n^{\circ} 71\right)$ et l'Itepeioc $\left(n^{\circ} 72\right)$ peuvent également être rapprochés du temple de Huitzilopochtli ${ }^{27}$, car c'est dans ces deux édifices que sont façonnées les effigies en pâte du dieu utilisées dans les rites de Toxcatl et de Panquetzaliztli ${ }^{28}$. Enfin, le lieu appelé Toxpalatl ( $\left.n^{\circ} 68\right)$, ce qui signifie « eau jaune », peut aussi être mis en rapport avec le temple de Huitzilopochtli. En effet, d'après le mythe 


\begin{tabular}{|c|c|c|c|c|}
\hline & $N^{\circ}$ liste & Complexe & Qualification Sahagún & Iteupan \\
\hline 1 & $\begin{array}{l}1 \text { à } 5 ; 13 ; \\
14(?) ; 41(?) \\
68(?) ; 71(?) \\
72(?) ; 74(?)\end{array}$ & $\begin{array}{l}\text { Pyramide double : Huitzilopochtli / } \\
\text { Tlaloc ; calmecac de Mexico ; Coacalco } \\
\text { (?); Toxpalatl (?); Tilocan (?); } \\
\text { Itepeioc (?); Atempan (?) }\end{array}$ & $\begin{array}{l}C u(3,4), \text { temple }(1), \text { maison }(4) \text {, } \\
\text { monastère }(13), \text { salle grillagée } \\
\text { comme une prison }(14) ; \text { tzompantli } \\
(41), \text { source }(68) \text {; maisons }(71,72 \text {, } \\
74)\end{array}$ & $\mathrm{x}$ \\
\hline 2 & $\begin{array}{l}6 ; 10 ; 11 ; \\
37 ; 39(?)\end{array}$ & Temple de Mixcoatl & $\begin{array}{l}\text { Cu (37), tzompantli (6), bosquet } \\
(10), \text { source (11); jeu de balle (39) }\end{array}$ & $\mathrm{x}$ \\
\hline 3 & $7 ; 12$ & $\begin{array}{l}\text { Temple des Seigneurs de la Mort } \\
\text { (Tlillan) }\end{array}$ & $C u(7)$, oratoire (12) & \\
\hline 4 & 8 & $\begin{array}{l}\text { Huey Quauhxicalco (Temple du } \\
\text { soleil ?) }\end{array}$ & Oratoire (8) & \\
\hline 5 & 9 & Temple des dieux du pulque (Tochinco) & $\mathrm{Cu}(9)$ & \\
\hline 6 & $15 ; 70(?)$ & Quauhxicalco de Tezcatlipoca & $C u(15)$; maison $(70)$ & \\
\hline 7 & 16 à 18 & $\begin{array}{l}\text { Temple de Xiuhtecuhtli (ou Temple de } \\
\text { Tezcatlipoca?) }\end{array}$ & $C u(16,17)$, tzompantli & \\
\hline 8 & $\begin{array}{l}19,20,21(?) \\
22,23(?), 24, \\
25,31 \text { à } 33 \\
73(?)\end{array}$ & $\begin{array}{l}\text { Temple de Huitznauac dédié à Omacatl } \\
\text { (ou à Tlacahuepan Cuexcotzin- } \\
\text { Tezcatlipoca?) }\end{array}$ & $\begin{array}{l}\mathrm{Cu}(19,25) \text {, oratoire }(20,22) \text {, } \\
\text { maison }(21) \text {, enclos }(23) \text {, } \\
\text { monastère }(24) \text {, source }(31) \text {, jeu de } \\
\text { balle }(32) \text {, tzompantli }(33) ; \text { maison } \\
(73)\end{array}$ & $x$ \\
\hline 9 & 26 & Temple de Macuilcipactli & $C u(26)$ & $x$ \\
\hline 10 & $27 ; 29$ & Temple de Tetlanman dédié à Chantico & $\mathrm{C} u$ (29), monastère (27) & \\
\hline 11 & 28 & Temple d'Iztac Cinteotl & $C u(28)$ & $\mathrm{x}$ \\
\hline 12 & 30 & Temple de Chicomecatl & $C u(30)$ & $\mathrm{x}$ \\
\hline 13 & 34 à 36 & Temple de Tlamatzincatl & $C u(34,36)$, monastère $(35)$ & \\
\hline 14 & 65,48 & Coatlan & $\begin{array}{l}\mathrm{Cu}(65) \text {, tzompantli (41), source } \\
(48)\end{array}$ & \\
\hline 15 & 43 & Temple de Chicomecoatl (=Cinteupan) & $C u(43)$ & $x$ \\
\hline 16 & 44 & $\begin{array}{l}\text { Temple des Centzontotochtin } \\
\left(=\text { Tochinco } n^{\circ} 9 \text { ?) }\right.\end{array}$ & $\mathrm{Cu}(44)$ & $x$ \\
\hline 17 & 45 & Temple de Cinteotl (=Cinteupan) & $C u(45)$ & $\mathrm{x}$ \\
\hline 18 & 46 et 47 & Temple de Quetzalcoatl-9 vent & $C u$ (47), partie d'un patio (46) & \\
\hline 19 & $49,52,56$ & Temple de Yiacatecuhtli & $\begin{array}{l}C u(52), \text { monastère (49), tzompantli } \\
(56)\end{array}$ & \\
\hline 20 & 50,53 & Temple de Huitzilinquatec & $C u(53)$, monastère $(50)$ & \\
\hline 21 & $51,54,55 ; 67$ & Temple de Yopico, dédié à Xipe & $C u(51)$, monastère ou oratoire & \\
\hline
\end{tabular}




\begin{tabular}{|l|l|l|l|c|}
\hline 22 & 57 & & $(54)$, tzompantli (55); maison (67) & \\
\hline 23 & 58 à $60 ; 66$ & Temple des Cihuateteo & $C u(57)$ & $\mathrm{X}$ \\
\hline 24 & 61,64 & Temple de Tzonmolco & $\begin{array}{l}\text { Oratoire }(58,60), \text { grotte ou cave } \\
(59), c u(66)\end{array}$ & \\
\hline 25 & 63 & Temple de Nappatecuhtli & $C u(64)$, monastère (61) & \\
\hline
\end{tabular}

\begin{tabular}{|l|l|l|l|l|}
\hline & & Edifices indépendants? & & \\
\hline 38 & Brasier de Nanahuatl et Xochcaye & Cave & Colonne & \\
\hline 40 & Ilhuicatitlan & Maison & \\
\hline 42 & Mecatlan & Maison & \\
\hline 69 & Tlacochcalco Quauhquiauac & Maison & \\
\hline 75 & Tlacochcalco de Tezcacoac & Maison & \\
\hline 76 & Grand calpulli d'Acatl Yiacapan & Petit $C u$ & \\
\hline
\end{tabular}

FIG. 4. - Recomposition des différents complexes cités dans la liste du Codex de Florence.

des pérégrinations, c'est sur une double caverne dans laquelle coulait de l'eau jaune et de l'eau bleue que les Mexicains virent l'oracle qui leur indiquait la fin de l'errance et, par la même occasion, le lieu de fondation de Tenochtitlan et du temple de Huitzilopochtli (Durán 1984, 2, pp. 45-47). Cependant, si on en croit Torquemada (1969, 2, p. 155), cette source aurait été redécouverte sur la «petite place du Marquis ${ }^{29}$, ce qui ne correspond pas aux abords immédiats de la pyramide principale.

Un ensemble d'édifices est dédié à Mixcoatl, le dieu de la chasse. Celui-ci comprend un temple de Mixcoatl ( $\left.\mathrm{n}^{\circ} 37\right)$, un tzompantli $\left(\mathrm{n}^{\circ} 6\right)$ destiné à recevoir les têtes des victimes sacrifiées en honneur du dieu, ainsi qu'un lieu de bain des prêtres, appelé Tlilapan $\left(\mathrm{n}^{\circ} 11\right)$. On peut également inclure dans ce groupe le Teutlalpan $\left(\mathrm{n}^{\circ} 10\right)$, le lieu duquel partait la grande procession du mois de Quecholli, vingtaine dédiée à Mixcoatl, et qui était peut-être une partie de la cour du temple de Mixcoatl transformée en zone de steppe à l'occasion de la fête. Enfin, le Teotlachco ( $\left.\mathrm{n}^{\circ} 39\right)$, le « terrain de jeu de balle divin » où des victimes appelées Amapantzitzin étaient mises à mort en Panquetzaliztli, peut aussi être rapproché de l'ensemble de Mixcoatl, en raison du lien mythique existant entre ce dieu et le jeu de balle, mais aussi du moment d'utilisation de cet édifice (Graulich 1980, p. 498). Cet édifice s'inscrit dans une série de huit lieux fréquentés à la fin de Quecholli ou au début de Panquetzaliztli (nº 34 à 41) (ibid., p. 505). Ceux-ci n'étaient cependant pas tous dédiés aux mêmes dieux, ce qui s'explique sans doute en partie par la pluralité des mythes réactualisés à cette occasion ${ }^{30}$.

Deux édifices peuvent être rapportés au culte des Seigneurs de la Mort, Mictlantecuhtli et Cihuacoatl ${ }^{31}$ : le Tlalxicco $\left(n^{\circ} 7\right)$ et le Tlillan Calmecac $\left(n^{\circ} 12\right)$. Le premier 
est présenté comme le lieu de sacrifice d'un personnificateur de Mictlantecuhtli, en Tititl. Les informateurs de Sahagún précisent que le prêtre de Tlillan venait y faire des offrandes d'encens. Ce prêtre de Tlillan était sans doute également spécialisé dans le culte de la déesse Cihuacoatl, car on sait, grâce à la liste, que les prêtres de cette déesse résident dans le Tlillan Calmecac $\left(\mathrm{n}^{\circ} 12\right)$ et, grâce à Durán $(1984,1, \mathrm{p} .131)$, que le sanctuaire situé au sommet du temple de Cihuacoatl était appelé Tlillan. Il semble donc que les prêtres de Cihuacoatl servaient également au culte de son divin époux, Mictlantecuhtli.

Un seul édifice de la liste du Codex de Florence, le Huey Quauhxicalco ( $\left.\mathrm{n}^{\circ} 8\right)$, est associé au culte du soleil. Montezuma lui-même y eflectuait le jeûne pour le soleil, tous les 260 jours, et des victimes appelées Chanchanme y étaient sacrifiées, en même temps que des personnificateurs du soleil et de la lune, et d'autres captifs. Durán (1984, 1, p. 106) signale un Temple du soleil, le Cuacuauhtin inchan, au sommet duquel se trouvait une pierre de sacrifice appelée cuauhxicalli sur laquelle mourait le personnificateur du soleil lors de la fête mobile du soleil, en 4 Mouvement. On connaît assez mal le déroulement de cette fête mobile, mais les concordances précises entre les deux descriptions permettent de croire que le Huey Quauhxicalco du Codex de Florence et le Cuacuauhtinchan de Durán sont un seul et même édifice dédié au culte solaire ${ }^{32}$.

L'édifice suivant, le Tochinco ( $\left.n^{\circ} 9\right)$, dont le nom signifie « lieu du lapin ", est dédié au culte des dieux du pulque ${ }^{33}$. Les informateurs de Sahagún indiquent qu'en Tepeilhuitl, on y sacrifiait une « image de Tochinco » ${ }^{34}$ que Sahagún identifie à un personnificateur d'Ome Tochtli. Le temple des Centzontotochtin $\left(n^{\circ} 44\right)$ est, lui aussi, dédié au culte des dieux du pulque. Cet édifice, qualifié d'iteupan, est décrit comme le lieu du sacrifice de personnificateurs de Tepuztecatl, de Totoltecatl et de Papaztac, lors de la fête de Tepeilhuitl. Il est possible que ces édifices ne forment qu'un seul ensemble cérémoniel. En effet, les Costumbres (1945, pp. 53-54) présentent le dieu Ome Tochtli comme le patron du village de Tepoztlan, ce qui revient à l'identifier à Tepuztecatl ${ }^{35}$, un des dieux du pulque célébré au temple des Centzontotochtin. Le lien entre ces deux édifices paraît donc très intime, bien qu'il ne puisse être précisé davantage.

Le Quauhxicalco $\left(n^{\circ} 15\right)$ est un édifice dédié à Tezcatlipoca, car il est présenté comme le lieu dans lequel Yiopoch, le nom que portent certains prêtres de Tezcatlipoca (López Austin 1965, p. 82), offre de l'encens. De plus, c'est dans un lieu lui aussi appelé quauhxicalco que le jeune homme incarnant Tezcatlipoca en vue de la fête de Toxcatl effectue des offrandes d'encens et joue de la flûte (Durán 1984, 1, p. 39). D'après le Codex de Florence (1950-1981, 2, p. 68), ce personnificateur était mis à mort dans un temple du même nom. Cependant, les descriptions physiques du lieu où celui-ci joue de la flûte ne permettent pas d'aflirmer qu'il s'agit du même édifice. Il se peut que le Tolnauac $\left(n^{\circ} 70\right)$, le théâtre de sacrifices qui avaient lieu lors du jour 1 Mort ${ }^{36}$, une journée dédiée au culte de Tezcatlipoca (Sahagún 1999, 4, p. 232), soit également associé au temple de ce dieu.

Les édifices suivants, un autre Quauhxicalco $\left(n^{\circ} 16\right)$, le Teccalco $\left(n^{\circ} 17\right)$ et un tzompantli ( $\left.\mathrm{n}^{\circ} 18\right)$, sont utilisés lors du sacrifice de captifs par le feu en Teotleco, une vingtaine dédiée au dieu du feu Xiuhtecuhtli, mais aussi à Tezcatlipoca ${ }^{37}$. Ainsi, c'est dans le premier lieu que danse un personnage écureuil, le techalotl, qui, par ses sifflements, indique le moment du sacrifice et c'est aussi là que l'arbre xocotl est dressé ${ }^{38}$. Le second édifice, le Teccalco ( $\left.n^{\circ} 17\right)$, est décrit comme le brasier sacrificiel, 
tandis que le troisième est le lieu d'exposition des têtes des victimes. Ces indications sont en adéquation avec la description de Teotleco dans le Codex de Florence ${ }^{39}$. Compte tenu du rôle du feu, destinataire des sacrifices en Teotleco et Xocotl Huetzi et du fait que, selon certaines indications, le temple de Xiuhtecuhtli comprenait un quauhxicalli, un tzompantli et un brasier (CF 1950-1981, 2, pp. 114-115), il n'est pas étonnant que, à propos du Quauhxicalli ( $\left.\mathrm{n}^{\circ} 16\right)$, les informateurs de Sahagún évoquent l'arbre xocotl en même temps que les sacrifices de Teotleco ${ }^{40}$. Sahagún (1999, p. 86) précise d'ailleurs clairement que l'arbre était dressé dans la cour du temple du dieu du feu ${ }^{41}$.

Une série d'édifices centrée sur le temple de Huitznauac $\left(n^{\circ} 19\right)$ peut être rapprochée du calpulli de Huitznauac, dont la divinité tutélaire n'est pas connue avec certitude. D'après la liste et la description de Panquetzaliztli dans le Codex de Florence (1950-1981,2, p. 148), des sacrifices de personnificateurs de Centzonhuitznaua avaient lieu au Huitznauac, en marge des sacrifices d'esclaves baignés qui se déroulaient dans le temple de Huitzilopochtli. Le temple de Huitznauac, dont l'existence est attestée par d'autres passages ${ }^{42}$, est souvent étroitement lié à Huitzilopochtli ${ }^{43}$, bien qu'un des édifices qui lui est associé, le calpulli de Huitznauac ( $\left.n^{\circ} 73\right)$, soit dédié plus spécifiquement à Tlacahuepan Cuexcotzin, un dieu qui, malgré son identité flexible, est particulièrement proche de Huitzilopochtli ${ }^{44}$.

Omacatl, qui n'est autre que Tezcatlipoca sous son aspect de dieu des banquets, a, lui aussi, un lien étroit avec les édifices associés au temple de Huitznauac où étaient effectués, en Panquetzaliztli, des sacrifices distincts de ceux qui avaient lieu au temple de Huitzilopochtli. Ce rapport est évoqué par une autre source de la vallée de Mexico, le Codex Magliabechiano (f. 36v), qui indique que le Huitznauac et le Tlacochcalcatl étaient des temples dédiés à Tezcatlipoca, mais aussi par le fait que Huitznauac est présenté comme le domaine d'Omacatl (CF 1950-1981, 1, p. 14) ${ }^{45}$.

Parmi les édifices pouvant être associés au Temple de Huitznauac, citons d'abord le Huitznauac Calmecac ( $\left.{ }^{\circ} 24\right)$, présenté comme le lieu de résidence des prêtres officiant dans ce temple. Le Quauhxicalco séparé $\left(n^{\circ} 25\right)$ est dédié à Omacatl, puisque les informateurs de Sahagún disent que le tzompantli du temple de Omacatl était " nourri » à cet endroit, ce qu'on peut interpréter comme l'endroit où les têtes décapitées étaient suspendues sur la plate-forme aux crânes. De plus, ils précisent qu'une statue en bois du dieu Omacatl qui se trouvait dans ce lieu était alimentée tous les 260 jours, sans doute lors de la fête mobile 2 Roseau (Ome Acatl). Sahagún indique que des statues représentant Omacatl étaient également conservées dans deux autres lieux : le Tezcacalco $\left(n^{\circ} 20\right)$ et le Teccizcalco $\left(n^{\circ} 22\right)$. Le Tezcatlachco ${ }^{46}\left(n^{\circ} 32\right)$ est le second édifice dédié à Omacatl dont le nom se rapporte au miroir (tezcatl). Il est présenté par les informateurs de Sahagún comme le terrain de jeu de balle sur lequel est mis à mort un personnificateur de Huitznauatl, les jours 2 Roseau (Ome Acatl). Le Tzompantli ( $\left.n^{\circ} 33\right)$ complète cette série de lieux, puisque des victimes appelées Omacame, ainsi que d'autres captifs, étaient mis à mort à cet endroit, à l'occasion d'une fète mobile. Il apparaît donc que ces édifices étaient très probablement dédiés à Omacatl, Tezcatlipoca sous son aspect de dieu des banquets. Deux édifices sont peut-être également associés à Omacatl, bien que les indices qui le suggèrent soient assez ténus : le Tezcaapan $\left(n^{\circ} 31\right)$, parce qu'il précède directement le Tezcatlachco $\left(\mathrm{n}^{\circ} 32\right)$ et que son nom évoque le miroir (tezcatl), comme deux édifices de 
ce groupe ; et le Huitztepehualco ( $\left.\mathrm{n}^{\circ} 23\right)$, l'endroit où les épines d'autosacrifice étaient dispersées - ce qui l'identifie peut-être à une cache cérémonielle ${ }^{47}$ —, en raison de sa position au milieu des édifices liés au calpulli de Huitznauac, et de leur étymologie commune ${ }^{48}$.

Les descriptions de plusieurs édifices qui sont apparemment sans rapport avec le dieu des banquets sont insérées entre les descriptions qui viennent d'être présentées. Trois de ces édifices sont définis comme des iteupan, ce qui permet de les identifier à des temples : ceux de Macuilcipactli ( $\left.n^{\circ} 26\right)$, d'Iztac Cinteotl $\left(n^{\circ} 28\right)$ et de Chicomecatl (n $\left.n^{\circ} 30\right)$.

La description du temple de Macuilcipactli ( $\left.n^{\circ} 26\right)$, « 5 Caïman », est le seul texte mentionnant ce dieu. Ce dieu « 5 » reste donc très mal connu.

À propos du temple d'Iztac Cinteotl ( $\left.\mathrm{n}^{\circ} 28\right)$, le dieu du maïs blanc, les informateurs de Sahagún indiquent qu'il était utilisé lors du jeûne pour le soleil et que c'est là qu'étaient mis à mort les captifs souffrant de problèmes de peau, les xixijoti ${ }^{49}$. Ce sacrifice n'est pas bien connu, mais on peut supposer qu'il avait quelque lien avec un des dieux associés aux maladies de peau, Xipe Totec et Tlaloc, tous deux d'ailleurs très proches de Cinteotl $^{50}$.

Les informateurs de Sahagún signalent que ce sacrifice était effectué au cours d'une fête mobile. Il est probable qu'il s'agit de la fête 1 Fleur, célébrant notamment la naissance du maïs (CF 1950-1981, 2, p. 238) ${ }^{51}$. En effet, la description de ce lieu est directement précédée et suivie des lieux utilisés à cette occasion : un temple dédié à Chantico, le Tetlanman ( $\left.\mathrm{n}^{\circ} 29\right)$, et son Calmecac ( $\left.\mathrm{n}^{\circ} 27\right)$. Leurs descriptions indiquent qu'une personnificatrice de Quaxolotl Chantico, la déesse du foyer ${ }^{52}$, était sacrifiée à cet endroit, après d'autres sacrifices. Comme le souligne Graulich (1980, pp. 700-701), plusieurs sources mentionnent des sacrifices conjoints dédiés à Xochipilli-Cinteotl et Cihuacoatl-Chantico. On peut dès lors croire que les « autres sacrifices » sont ceux qui étaient effectués dans le temple d'Iztac Cinteotl et peut-être même ceux du temple de Macuilcipactli, puisqu'en tant que dieu « 5 », ce dieu était étroitement lié à XochipilliCinteotl.

L'édifice suivant, le temple de Chicomecatl (7 Vent ?) ${ }^{53}$ (nº 30 ) est lui aussi le théâtre de sacrifices nocturnes liés à la fête 1 Fleur. Les quelques indications conservées à propos de cette fête ne permettent pas de préciser la personnalité de cette divinité, qui porterait le nom-calendrier de Quetzalcoatl lorsqu'il créa l'humanité à partir de cendres (Codice Chimalpopoca 1945, p. 5) ${ }^{54}$.

Ces cinq descriptions renverraient donc à quatre ensembles cérémoniels différentsfréquentés lors de la fête mobile 1 Fleur : ceux de Macuilcipactli, de Tetlanman, d'Iztac Cinteotl et de Chicomecatl.

Vient ensuite l'ensemble de lieux dédiés à Tlamatzincatl, un des dieux du pulque que Sahagún (1999, p. 88 et p. 141) identifie à Tezcatlipoca. Un personnificateur de ce dieu est sacrifié à la fin de Quecholli, dans son temple, au moment de la mise à mort des personnificateurs de Mixcoatl et de Cihuacoatl dans leur temple respectif (CF 19501981, 2, p. 139). L'existence d'un temple distinct est corroborée par la liste de Sahagún, puisqu'un temple $\left(\mathrm{n}^{\circ} 34\right)$, un calmecac $\left(\mathrm{n}^{\circ} 35\right)$ et un quauhxicalco $\left(\mathrm{n}^{\circ} 36\right)$ sont associés, plus ou moins précisément, au sacrifice du personnificateur de Tlamatzincatl. Il faut également souligner que le nom du temple, Tlamatzinco, est également celui d'un des calpulli de la ville. 
Deux édifices sont qualifiés de Cinteupan, de temple du maïs. Le premier $\left(n^{\circ} 43\right)$ est associé à Chicomecoatl, puisque c'est là qu'avaient lieu, en Ochpaniztli, le sacrifice et l'écorchement d'une personnificatrice de la déesse ${ }^{55}$. Un temple de Chicomecoatl ${ }^{56}$ est également mentionné dans les descriptions de Huey Tozoztli récoltées par Sahagún (PM 1997, p. 58 ; CF 1950-1981, 2, p. 63). Le second édifice ( $\left.n^{\circ} 45\right)$ était probablement dédié à Cinteotl, puisqu'un personnificateur du dieu était sacrifié en ce lieu. Ce sacrifice n'est connu que par le commentaire laconique de la liste. Vu les maigres indications concernant cet édifice, il est prudent de considérer ces deux lieux comme des ensembles cérémoniels différents.

Seule la liste du Codex de Florence indique que, lors de la fête de Atl Caualo ${ }^{57}$, des sacrifices étaient pratiqués en honneur de Chiconauh Ehecatl, " 9 Vent », qui n'est autre que Quetzalcoatl sous son aspect de dieu du vent ${ }^{58}$. Le Netotiloyan ( $\left.n^{\circ} 46\right)$ et le Chililico $\left(n^{\circ} 47\right)$ sont décrits comme le théâtre de ces rites : les esclaves baignés destinés à être sacrifiés ainsi qu'un personnificateur du dieu dansaient dans le premier endroit et étaient mis à mort dans le second, pendant la nuit. D'après la liste des prêtres (CF 1950-1981, 2, pp. 207-208), c'est le prêtre appelé Molonco qui était chargé de l'organisation des sacrifices en honneur de ce dieu. Le nom de ce prêtre renvoie probablement au calpulli du même nom Molonco, tandis qu'un calpulli appelé Chilililo est repris dans certaines listes recueillies par Tezozomoc $(1878,70$, p. 515).

Un groupe d'édifices est associé à la fin de Quecholli et à Coatlicue. Il est composé d'une source, le Coaapan ( $\left.\mathrm{n}^{\circ} 48\right)$, et d'un édifice, probablement un temple, le Coatlan ( $n^{\circ}$ 65). Dans la liste du Codex de Florence, le premier est décrit comme le lieu de bain du seul prêtre de Coatlan et le second comme le lieu de mise à mort des Centzonhuitznaua, " lorsqu'on forait ou lorsque Quecholli se terminait » (CF 1950-1981, 2, p. 191). Un autre sacrifice avait lieu sur le Coatlan, à la fin de Quecholli, celui de la personnificatrice de Coatlicue (ibid., p. 140), la déesse vénérée par les habitants du calpulli de Coatlan (ibid., p. 57).

Différents édifices sont dédiés au dieu des marchands, Yiacatecuhtli : le temple de Yiacatecuhtli ( $\left.n^{\circ} 52\right)$, où était sacrifié un personnificateur du dieu en Tititl ; le Pochtlan ( $\left.n^{\circ} 49\right)$, qui est décrit comme le lieu de résidence des prêtres de ce même temple et un tzompantli $\left(\mathrm{n}^{\circ} 56\right)$, sur lequel étaient disposées les têtes des victimes sacrifiées dans le temple en Xocotl Huetzi. Ce dieu, patron de la confrérie des marchands, est intimement lié au calpulli de Pochtlan ${ }^{59}$, ce qui se traduit ici dans le nom du calmecac de ce dieu. Les marchands offraient des esclaves en sacrifices pour certaines fềtes annuelles, notamment en Panquetzaliztli (CF 1950-1981, 9, p. 63) et Xocotl Huetzi (Durán 1984, 1, p. 120). Les sources ne mentionnent toutefois pas le sacrifice du personnificateur évoqué dans la description du temple.

Le temple de Huitzilinquatec ( $\left.n^{\circ} 53\right)$ et l'Atlauhco $\left(n^{\circ} 50\right)$ sont deux édifices liés au culte d'une déesse uniquement connue par la liste des édifices : Huitzilinquatec. Cette déesse était personnifiée par une femme qui était sacrifiée en Tititl. Elle disposait de son propre clergé, qui résidait dans l'édifice appelé Atlauhco $\left(\mathrm{n}^{\circ} 50\right)$. Encore une fois, le nom de ce calmecac renvoie directement à un calpulli de la ville. Un autre lieu de la liste $\left(n^{\circ} 60\right)$ porte également ce nom. Il est, lui, utilisé en Ochpaniztli, pour le sacrifice d'une déesse, elle aussi pratiquement inconnue, Atlauhco cihuateotl (voir infia).

On sait, d'après les descriptions des fêtes de l'année solaire, qu'un temple situé à l'intérieur de l'enceinte cérémonielle était dédié à Xipe Totec : le Yopico ${ }^{60}$. Ce temple, 
mais aussi le calmecac et le tzompantli qui en dépendent, sont signalés dans la liste. Le premier ( $\left.\mathrm{n}^{\circ} 51\right)$, utilisé lors de la fête de Xipe en Tlacaxipehualiztli, est décrit comme le lieu de sacrifice de Mayahuel, la déesse de l'agave, et de Tequitzin, un dieu mal connu ${ }^{61}$. Les sacrifices d'esclaves en Tlacaxipehualiztli sont également évoqués par Durán (1984, 1, p. 96), qui indique que chaque quartier de la ville offrait un esclave personnifiant son dieu patron. Il cite quelques-uns de ces dieux, parmi lesquels on retrouve Mayahuel, ce qui confirme les informations de la liste. Le Calmecac de Yopico ( $\left.n^{\circ} 54\right)$ est lui aussi décrit comme un lieu de sacrifices nocturnes effectués au cours de la même vingtaine, sans plus de précision ${ }^{62}$. Le Tzompantli ( $\left.\mathrm{n}^{\circ} 55\right)$ du même ensemble est logiquement présenté comme l'endroit où sont conservées les têtes des personnes sacrifiées au cours de la vingtaine dédiée à Xipe. Le Yopicalco $\left(n^{\circ} 67\right)$ est décrit comme le lieu où étaient reçus les seigneurs de l'Anahuac, venus de l'étranger. Cet édifice est peut-être à rapprocher du temple de Yopico, en raison de l'étymologie de son nom et du lien qui unit Xipe Totec à la région pacifique, appelée Anahuac ${ }^{63}$.

Macuilmalinalli et Topantlacaqui ${ }^{64}$, les dieux auxquels était dédié le Temple de Macuilmalinalli ( $n^{\circ} 57$ ) ne sont pas connus par ailleurs. Les informateurs de Sahagún affirment qu'ils étaient honorés par des sacrifices lors de la Fête des Fleurs, Xochilhuitl, qui est désignée aussi comme la fète de Xochipilli (Sahagún 1999, p. 40) et de Xochiquetzal (Durán 1984, 1, pp. 151-152), deux patrons des arts. Si l'identité des deux dieux honorés dans cet édifice ne peut être précisée davantage, des sacrifices en leur honneur s'intègrent bien dans la fête de Xochipilli, car celui-ci est étroitement lié aux dieux $« 5 » 65$.

La liste se poursuit avec différents édifices dédiés aux Cihuateteo, les «femmes divines ${ }^{66}$. Le premier, l'Aticpac ( $\left.n^{\circ} 58\right)$, est présenté comme un lieu de sacrifice en honneur de ces déesses, le jour 7 Serpent. D'après la liste des prêtres, une personnificatrice d'une déesse appelée Aticpaccalqui cihuatl, « Femme de la maison sur l'eau », était sacrifiée et écorchée et un prêtre revêtu de sa peau parcourait les rues, une caille entre les dents ${ }^{67}$. Ces indications, ainsi que l'étymologie du nom de la divinité ont permis de l'identifier comme une des déesses de l'eau sacrifiées en Ochpaniztli en même temps que Toci ${ }^{68}$ (Graulich 1980, p. 381). Le lieu appelé Atlauhco ( $\left.{ }^{\circ} 60\right)$, est lui aussi le théâtre du sacrifice d'une divinité inconnue, mais dont le nom, Atlauhco cihuateotl, « la déesse du lieu de la fondrière » ${ }^{69}$, indique qu'il s'agit aussi d'une des déesses de l'eau sacrifiées lors de la fête du Balayage. Le Netlatiloyan ( $\left.n^{\circ} 59\right)$ est décrit comme l'endroit où sont enfouies les peaux des personnifications de Cihuateteo sacrifiées au Xochicalco $\left(\mathrm{n}^{\circ} 66\right)$ en Ochpaniztli, ce qui l'identifie sans doute à une cache cérémonielle. En Tlacaxipehualiztli, l'autre vingtaine où les victimes étaient écorchées, les peaux usagées étaient enfouies dans une cache située à l'intérieur du Yopico ${ }^{70}$. On peut donc supposer que la cache Netlatiloyan se trouvait, elle aussi, à proximité du temple où les victimes étaient sacrifiées, le Xochicalco $\left(n^{\circ} 66\right)$. Dans la description de ce dernier; les informateurs de Sahagún mentionnent le sacrifice et l'écorchement de différents personnificateurs, en Ochpaniztli. Une de ces victimes personnifie la déesse de l'eau Atlatonan, tandis que les deux autres représentent des divinités du maïs, Itzac Cinteotl (Cinteotl blanc) et Tlatlauhqui Cinteotl (Cinteotl rouge) ${ }^{71}$.

Deux édifices, le Tzonmolco $\left(\mathrm{n}^{\circ}\right.$ 64) et le Tzonmolco calmecac $\left(\mathrm{n}^{\circ} 61\right)$, forment un ensemble dédié au dieu du feu, fréquenté au cours de la vingtaine d'Izcalli. Le 
Tzonmolco est décrit comme le lieu de sacrifice, en Izcalli, de quatre personnificateurs de Xiuhtecuhtli ${ }^{72}$, ainsi que celui des Ilhuipapaneca et des Temilolca ${ }^{73}$. Deux femmes étaient également mises à mort : Cihuatontli, qui peut probablement être identifiée à la femme de Xiuhtecuhtli (Graulich 1980, p. 561), et Nancotlaceuhqui ${ }^{74}$, une déesse mal connue. Le Codex de Florence dit que les différents Xiuhtecuhtli étaient personnifiés par des esclaves baignés et que les Ilhuipapaneca et les Temilolca étaient des captifs, sacrifiés au préalable pour que les esclaves soient ensuite mis à mort sur leurs corps. Le Tzonmolco calmecac $\left(\mathrm{n}^{\circ} 61\right)$ est, quant à lui, présenté comme l'endroit où Montezuma prenait le feu pour encenser, lors de Huauhquiltamalcualiztli, le dixième jour d'Izcalli. Sahagún ajoute que c'était le lieu de résidence des prêtres de Xiuhtecuhtli ${ }^{75}$. Bien qu'il soit clairement dédié au dieu du feu, on ne peut pas déterminer si l'ensemble Tzonmolco, sans doute associé au calpulli du même nom, se confond avec l'ensemble utilisé en Xocotl Huetzi et Teotleco qui a été identifié plus haut.

Le dieu Nappatecuhtli ( $\left.n^{\circ} 63\right)$, le patron des fabricants de nattes, était honoré dans un temple sur pyramide où, disent les informateurs de Sahagún, un personnificateur du dieu était sacrifié en Tepeilhuitl. Ce sacrifice n'est pas mentionné par d'autres sources, mais sa place au sein de la vingtaine est entièrement justifiée, puisqu'il est considéré comme un des Tlaloque, les dieux de la pluie à l'honneur en Tepeilhuitl ${ }^{76}$.

\section{Les édifices ne pouvant être rattachés à un ensemble particulier}

Faute de données complémentaires, certains édifices de la liste ne peuvent pas être rattachés précisément à l'un des 25 ensembles repérés ci-dessus. Il serait toutefois étonnant qu'il s'agisse d'édifices totalement indépendants.

Malgré les liens évidents qui l'unissent aux édifices qui le précèdent et qui sont fréquentés en Quecholli et en Panquetzaliztli, on ne peut déterminer à quel ensemble le Netlatiloyan $\left(\mathrm{n}^{\circ} 38\right)$ a pu être rattaché. Il est probable que ce brasier ${ }^{77}$, théâtre du sacrifice des personnificateurs de Nanahuatl et de Xochcuaye, ce qui renvoie clairement à celui qui a permis la naissance du soleil et de la lune à Teotihuacan (Graulich 1980, p. 505), a été situé à proximité des temples de Mixcoatl et/ou du Grand Temple, où se déroulaient les principaux rites de ces vingtaines. Le lieu Ilhuicatitlan $\left(\mathrm{n}^{\circ} 40\right)$, qui est lui aussi inséré dans cette succession de 8 édifices et qui est présenté comme le lieu des sacrifices liés à un lever héliaque de Vénus, ne peut pas non plus être associé précisément à un de ces temples, d'autant qu'il n'était pas fréquenté lors de ces vingtaines, mais lors d'une fète mobile qui se tenait sans doute tous les 584 jours.

Le Mecatlan ( $\mathrm{n}^{\circ}$ 42) était peut-être un édifice associé à un calmecac, puisqu'il est décrit comme l'endroit où les prêtres venaient jouer des instruments de musique, des trompes, précise Sahagún. Cependant, on ne peut déterminer s'il est associé à un ensemble cérémoniel particulier ou si, au contraire, c'était un lieu d'exercice à l'usage de l'ensemble du clergé, puisqu'on sait que le jeu de trompe était intimement lié à la pénitence que pratiquaient tous les prêtres.

La question de l'association des trois tlacochcalco de la liste à différents ensembles reste problématique. D'après leur étymologie et Torquemada (1969, 2, p. 146), ils peuvent être identifiés aux « salles d'armes » associées aux portes de l'enceinte ${ }^{78}$, ce qui n'implique pas nécessairement qu'ils soient totalement indépendants. De plus, certains lieux appelés tlacochcalco sont présentés comme étant liés au dieu Tezcatli- 
poca ${ }^{79}$. Cependant, pour compliquer le tout, les trois tlacochcalco de la liste ne se rapportent pas clairement au dieu "miroir fumant ». Ainsi, malgré l'existence de certains indices, et comme le souligne Olivier (1997, p. 194), le Tlacochcalco Acatl Yiacapan $\left(n^{\circ} 21\right)$ ne peut être identifié avec certitude à un édifice dédié à Tezcatlipoca, car le Grand Calpulli Acatl Yiacapan ( $\left.\mathrm{n}^{\circ} 76\right)$ est clairement présenté comme un lieu dédié aux dieux de la pluie. Le second, le Tlacochcalco Quauhquiauac $\left(\mathrm{n}^{\circ} 69\right)$, est présenté comme un lieu de sacrifice à Macuiltotec, un dieu qui n'est pas connu par ailleurs, mais qui fait sans doute partie, en tant que dieu « 5 », des MimixcoaHuitznaua sacrifiés en Panquetzaliztli (Graulich 1980, p. 513) ${ }^{80}$. Enfin, le Tlacochcalco de Tezcacoac ${ }^{81}\left(n^{\circ} 75\right)$ est un lieu qui n'est mentionné que dans la liste d'édifices et qui y est décrit comme un arsenal servant occasionnellement de lieu de sacrifice de captifs. Il était peut-être associé à une déesse de la terre peu connue, Tezcacoac Ayopechtli, évoquée dans les Primeros Memoriales (Seler 1992, 2, p. 261). Ainsi donc, les données variées concernant les différents tlacochcalco ne permettent pas de les associer à un complexe précis, et encore moins à un temple de Tezcatlipoca. Mais faut-il pour autant en conclure que ces arsenaux étaient totalement indépendants ?

L'Atempan ( $\left.\mathrm{n}^{\circ} 74\right)$ est décrit comme le lieu de rassemblement des tlacateuhti ${ }^{82}$, avant qu'ils ne soient emmenés vers la lagune ou certains sommets de montagne pour y être sacrifiés. Les informations très précises contenues dans la description nahuatl concordent avec la description de la vingtaine d'Atl caualo, dédiée à Tlaloc. Sahagún précise que ces victimes sont des enfants et des "lépreux» appelés xixioti, ce qui renvoie aux victimes sacrifiées au Temple du Cinteotl Blanc $\left(\mathrm{n}^{\circ} 28\right)$. Cet édifice pourrait donc être associé au temple de Tlaloc, d'autant que son nom peut se traduire par « sur la rive » (Garibay 1999, p. 917), et/ou à un temple du maïs ${ }^{83}$, mais aussi, ce qui s'explique plus difficilement, au temple dédié à la déesse Toci, qui est plusieurs fois appelé Atempan ${ }^{84}$.

Le Techielli ( $\left.\mathrm{n}^{\circ} 77\right)$, qui est présenté comme un lieu de conservation des branches de pin (acxoyatl), ne peut pas être mis en rapport avec un ensemble cérémoniel particulier, tant l'usage de ces branches est fréquent et généralisé.

À la fin de la liste, le Grand Calpulli d'Acatl Yiacapan ( $\left.n^{\circ} 76\right)$ est décrit comme l'endroit où étaient choisis, sacrifiés, démembrés et cuits les personnificateurs des Tlaloque. Les informateurs de Sahagún précisent qu'ils étaient ensuite mangés, uniquement par les seigneurs. Le sacrifice de ces victimes n'est pas évoqué par ailleurs, mais il est très probable que cet édifice était associé au temple de Tlaloc, bien que, comme le suggère Olivier (1997, p. 194), le lieu appelé Acatliyacapan puisse aussi avoir eu un lien avec le culte de Tezcatlipoca.

Les informateurs de Sahagún terminent leur liste par une évocation des calpulli. Ils les présentent comme « toutes les petites maisons sur le pourtour et qui entouraient les temples $"{ }^{85}$, dans lesquelles les seigneurs et les valeureux guerriers jeûnaient tous les 20 jours. Il semble donc que ces lieux de pénitence, associés à des temples que la liste n'identifie pas, dépendaient des différents quartiers de la ville ${ }^{86}$. 


\section{CONCLUSION}

\section{Le nombre d'édifices}

Quelque 25 ensembles cérémoniels peuvent donc être dégagés des 78 descriptions de la liste (voir Figure 4). Ils comprennent un nombre plus ou moins important de structures, sont dédiés à une divinité particulière et, par conséquent, étaient généralement fréquentés lors des mêmes fêtes, et peuvent souvent être mis en rapport avec un des calpulli de la ville de Mexico. Ce regroupement des descriptions en différents ensembles permet de ramener le nombre de structures évoquées par la liste de Sahagún à des proportions plus proches de celles des autres chroniqueurs, qui ne comptabilisent pas les bâtiments dépendant des temples. Bon nombre des « édifices » de la liste sont, en effet, des endroits non construits ou peu aménagés, comme des sources, ou sont des constructions de petite taille. En plus de mieux correspondre aux autres descriptions de l'enceinte, la répartition des 78 édifices en ensembles composés de structures de taille très variable concorde avec les données matérielles récoltées par les archéologues dans le centre de Mexico, puisque, rien que sur la plate-forme entourant la pyramide double, au moins quatre petites structures ont été mises au jour.

\section{Les divinités auxquelles sont dédiés les édifices}

En examinant la liste en fonction des dieux, on peut retrouver les différents temples sur pyramide mentionnés par Durán même si, dans certains cas, cette identification reste hypothétique (voir Figures $2 \mathrm{a} \& 2 \mathrm{~b}$ ). Il apparaît donc que les temples des divinités principales du panthéon aztèque sont effectivement évoqués dans la liste. Cependant, on ne peut que s'étonner du caractère discret de cette évocation, puisque les édifices dédiés à des dieux aussi centraux que Quetzalcoatl ou Tezcatlipoca sont, pour le premier, deux structures vouées au culte de son aspect de dieu du vent $\left(n^{\circ} 46\right.$ \& 47 ) et, pour le second, un oratoire (quauhxicalco) isolé $\left(\mathrm{n}^{\circ} 15\right)$, ainsi que différents groupes d'édifices honorant un aspect très particulier du dieu (Omacatl ; Tlamatzincatl) toujours étroitement liés à un des calpulli de la ville, et peut-être certains tlacochcalco $\left(\mathrm{n}^{\circ} 69 \& 75\right)$.

Mais on a aussi pu montrer que, dans certains cas, la liste de Sahagún complète bien les indications de Durán. C'est notamment le cas du temple de Xiuhtecuhtli, dont on avait souligné l'absence dans le Libro de los ritos de Durán, et qui se cache sans doute derrière les édifices de l'ensemble 7 (nº 16 à 18) (voir Figure 4).

Cependant, même si une dizaine de lieux sont dédiés, plus ou moins directement, aux principales divinités aztèques, la plupart des autres édifices sont associés à des divinités peu ou mal connues. En outre, les sacrifices qui y sont accomplis se révèlent souvent être des rites mineurs, dont on ne trouve pas toujours d'écho en dehors de ce chapitre précis du Codex de Florence. Pour comprendre le caractère inédit et parfois insolite de ces données, il faut avant tout souligner le fait qu'une petite quinzaine de descriptions renvoie directement aux fêtes mobiles, les fêtes du calendrier divinatoire. Or, les cérémonies liées à ces fêtes sont particulièrement mal connues, tant les informations conservées au sujet de ce calendrier sont rares et incomplètes. Il ne faut donc pas s'étonner que les sacrifices succinctement évoqués dans cette liste ne soient pas 
décrits ailleurs. Au contraire, leur mention est précieuse, car il s'agit d'une des rares sources présentant certains des nombreux rites qui émaillaient le cycle divinatoire de 260 jours et le cycle vénusien. Dans cette perspective, il est probable qu'un grand nombre de divinités nous resteront inconnues, puisqu'il apparaît que des divinités très particulières étaient mises à l'honneur lors des fêtes mobiles et que bon nombre de ces fêtes ne sont pas même évoquées dans les autres sources. Ainsi donc, le fait qu'un certain nombre d'édifices soient utilisés lors des fètes mobiles, et notamment tous les lieux qualifiés d'iteupan (excepté le temple de Mixcoatl et celui de Nappatecuhtli), permet de comprendre pourquoi la personnalité des dieux auxquels ils sont dédiés Omacatl, Macuilcipactli, Chantico, Itzac Cinteotl, Chicomecatl, Chiconauhehecatl, Macuilmalinalli - reste souvent mal connue.

En ce qui concerne les divinités associées aux lieux utilisés lors des fètes des vingtaines, elles ne sont pas non plus toutes connues et les sacrifices qui leur étaient dédiés semblent également secondaires. Cependant, la validité des informations de la liste peut être confirmée, soit par les autres descriptions des vingtaines, qui évoquent, sans pour autant les décrire, d'autres sacrifices en marge des sacrifices principaux, soit par le fait que les sacrifices mentionnés par la liste s'inscrivent généralement dans la logique symbolique de la fête, comme c'est le cas, par exemple, pour le sacrifice de Nappatecuhtli $\left(n^{\circ} 63\right)$. Le fait que ce document confirme ou complète les autres sources à propos des sacrifices des vingtaines renforce donc le crédit à apporter à la liste en général, mais surtout aux données concernant les fêtes mobiles.

Les nombreuses références aux calpulli contenues dans la liste permettent peutêtre d'expliquer pourquoi les dieux qui y sont évoqués sont si peu connus ou, dans le cas des dieux principaux, pourquoi ils sont honorés sous un de leurs aspects particuliers. En effet, l'analyse de la liste permet de rapprocher tous les ensembles de descriptions - à l'exception des premiers ensembles cérémoniels dédiés aux dieux principaux et des édifices qualifiés de iteupan - à un des quartiers de la ville (Figure 5). Les différents dieux qu'honorait chaque calpulli ne sont pas tous bien connus, car la documentation au sujet de ces groupes sociaux et de leurs pratiques rituelles est très inégale. Comme la liste évoque les dieux des 15 calpulli les plus anciens ${ }^{87}$, ainsi que ceux d'autres calpulli moins communs, il est possible que les informateurs de Sahagún aient, en composant la liste, présenté les édifices en fonction des différents quartiers de la ville qui en avaient la charge. Cette hypothèse permettrait notamment d'expliquer la place prépondérante d'Omacatl par rapport aux autres divinités principales présentées dans la première partie de la liste, puisqu'on sait que le calpulli de Huitznauac, dont il est le patron, jouait un rôle majeur dans les rites de Panquetzaliztli. De manière plus générale, cette interprétation fait apparaître que la relative méconnaissance des dieux vénérés dans les lieux de la liste est probablement due à la nature même de ces divinités, car ce sont des divinités tutélaires propres à une profession déterminée et à un quartier particulier.

\section{La composition de la liste et sa ou ses références externes}

Si ces indications permettent de comprendre pourquoi les dieux cités dans la liste sont si peu connus, elles n'expliquent pas pour autant les liens qui unissent les 


\begin{tabular}{|c|c|c|c|c|c|}
\hline & Lieu & Dieu & Fête & Jour & Calpulli \\
\hline 1 & Temple de Huitzilopochtli & Huitzilopochtli & & & \\
\hline 2 & Tlalocan, temple d'Epcoatl & Tlaloc & Etzalqualiztli & & \\
\hline 3 & Macuilcalli ; Macuilquiauitl & & & 5 Maison? & \\
\hline 4 & Teccizcalli & & & & \\
\hline 5 & Poyauhtlan & & Etzalqualiztli & & \\
\hline 6 & Tzompantli de Mixcoatl & Mixcoatl & & & \\
\hline 7 & Tlalxicco & & Tititl & & \\
\hline 8 & Huey Quauhxicalco & Soleil & & & \\
\hline 9 & Tochinco & dieu du pulque & Tepeilhuitl & & \\
\hline 10 & Teotlalpan & Mixcoatl & Quecholli & & \\
\hline 11 & Tlilapan & & & & Chalmecah \\
\hline 12 & Tlillan calmecac & Cihuacoatl & & & \\
\hline 13 & Calmecac de Mexico & Tlaloc & & & Mexico? \\
\hline 14 & Coacalco & & & & \\
\hline 15 & Quauhxicalco & Tezeatlipoca & Toxcatl & & \\
\hline 16 & Autre Quauhxicalco & $?$ & Teotl Eco & & \\
\hline 17 & Tecealco & & & & \\
\hline 18 & Tzompantli & & & & \\
\hline 19 & Temple de Huitznauac & & Panquetzaliztli & & Huitznauac \\
\hline 20 & Tezcacalco & Omacatl & & Jour non fixe & \\
\hline 21 & Tlacochcalco Acatl Yiacapan & $\begin{array}{l}\text { [Tezcatlipoca? } \\
\text { Tlaloc ?] }\end{array}$ & & & Acatliacapan? \\
\hline 22 & Teccizcalco & Omacatl & & & \\
\hline 23 & Huitztepehualco & I & & & \\
\hline 24 & Calmecac de Huitznahuac & Omacatl & & & Huitznauac \\
\hline 25 & Quauhxicalco séparé & & & 2 Roseau & \\
\hline 26 & Temple de Macuilcipactli & Macuilcipactli & & 5 Crocodile? & \\
\hline 27 & Calmecac de Tetlanman & Chantico & & & Cihuatecpac? \\
\hline 28 & Temple de Iztac cinteotl & Iztac Cinteotl & & 4 Mouvement & \\
\hline 29 & Tetlanman & Chantico & & 1 Fleur & Cihuatecpac? \\
\hline 30 & Temple de Chicomecatl & Chicomecatl & & 1 Fleur & \\
\hline
\end{tabular}




\begin{tabular}{|c|c|c|c|c|c|}
\hline 31 & Tezcaapan & I & & & Huitznauac \\
\hline 32 & Tezcatlachco & Omacatl & & & \\
\hline 33 & Tzompantli & & & & \\
\hline 34 & Tlamatzinco & Tlamatrincatl & & & Tlamatzinco \\
\hline 35 & Tlamatzinco calmecac & & & & \\
\hline 36 & Quauhxicalco & & & & \\
\hline 37 & Mixcoateupan & Mixcoatl & fin Quecholli & & \\
\hline 38 & Netlatiloyan & Soleil/Lune & $\begin{array}{l}\text { [début } \\
\text { Panquetzaliztli] }\end{array}$ & & \\
\hline 39 & Teotlacheo & Mixcoatl? & Panquetzaliztli & & \\
\hline 40 & Ilhuicatitlan & Venus & $\begin{array}{l}\text { [début } \\
\text { Panquetzaliztli] }\end{array}$ & & \\
\hline 41 & Grand tzompantli & Huitzilopochtli & Panquetzaliztli & & \\
\hline 42 & Mecatlan & & & & \\
\hline 43 & Temple du maĩs & dieu du maĩs & Ochpaniztli & & \\
\hline 44 & Temple des Centzontotochtin & dieu du pulque & Tepeilhuitl & & \\
\hline 45 & Temple du maiss & dieu du maĩs & & & \\
\hline 46 & Netotiloyan & 9 vent & Atl caualo & & [Molonco] \\
\hline 47 & Chililico & 9 vent & & 9 Vent & Chililico \\
\hline 48 & Coaapan & & & & Coatlan \\
\hline 49 & Pochtlan & Yiacatecuhtli & [Tititl] & & Pochtlan \\
\hline 50 & Atlauhco & Huitzilinquatec & & & Atlauhco \\
\hline 51 & Yopico & [Xipe Totec] & Tlacaxipehualiztli & & Yopico \\
\hline 52 & Temple de Yacatecuhtli & Yiacatecuhtli & Tititl & & [Pochtlan] \\
\hline 53 & Temple de Huitzilinquatec & Huitzilinquatec & & & [Atlauhco] \\
\hline 54 & Calmecac de Yopico & [Xipe Totec] & Tlacaxipehualiztli & & Yopico \\
\hline 55 & Tzompantli de Yopico & [Xipe Totec] & Tlacaxipehualiztli & & Yopico \\
\hline 56 & Tzompantli & Yiacatecuhtli & Xocotl Huetzi & & [Pochtlan] \\
\hline 57 & Temple de Macuilmalinalli & dicu "5" & & Xochilhuitl & \\
\hline 58 & Aticpac & & [Ochpaniztli ?] & 7 Serpent & \\
\hline 59 & Netlatiloyan & Cihuateteo & Ochpaniztli & & \\
\hline 60 & Atlauhco & Cihuateteo & & & $\begin{array}{l}\text { Atlauhco / } \\
\text { Coatlan }\end{array}$ \\
\hline
\end{tabular}




\begin{tabular}{|c|c|c|c|c|c|}
\hline 61 & Tzonmolco calmecac & Xiuhtecuhtli & Izcalli & & Tzonmolco \\
\hline 62 & Temalacatl & [Xipe Totec] & Tlacaxipehualiztli & & Yopico? \\
\hline 63 & Temple de Nappatecuhtli & Nappatecuhtli & Tepeilhuitl & & \\
\hline 64 & Tzonmolco & Xiuhtecuhtli & Izcalli & & Tzonmolco \\
\hline 65 & Coatlan & Huitzilopochtli & fin Quecholli & & Coatlan \\
\hline 66 & Xochicalco & $\begin{array}{l}\text { dieu du maïs / } \\
\text { Cihuatcotl }\end{array}$ & Ochpaniztli & & Xochicalco \\
\hline 67 & Yopicalco & [Xipe Totec ?] & & & Yopico? \\
\hline 68 & Toxpalatl & & & & \\
\hline 69 & Tlacochcalco quauhquiahuac & Tezcatlipoca? & $\begin{array}{l}\text { feu nouveau/ } \\
\text { Panquetzaliztli/ } \\
\text { Tlacaxipehualiztli }\end{array}$ & & $\begin{array}{l}\text { Tlacochcalco / } \\
\text { Cuauhquiahuac }\end{array}$ \\
\hline 70 & Tolnauac & Tezcatlipoca & & 1 Mort & \\
\hline 71 & Tilocan & Huitzilopochtli & $\begin{array}{l}\text { [Panquetzaliztli- } \\
\text { Toxcatl] }\end{array}$ & & \\
\hline 72 & Itepeioc & & $\begin{array}{l}\text { [Panquetzaliztli- } \\
\text { Toxcatl] }\end{array}$ & & \\
\hline 73 & Calpulli de Huitznauac & $\begin{array}{l}\text { Tlacahuepan } \\
\text { Cuexcotzin }\end{array}$ & Panquetzaliztli & & Huitznauac \\
\hline 74 & Atempan & [Tlaloc] & & & Natempan \\
\hline 75 & Tlacochcalco Tezcacoac & $\begin{array}{l}\text { [Tlaloc] / } \\
\text { Tezcatlipoca? }\end{array}$ & & & $\begin{array}{l}\text { Tlacochcalco/ } \\
\text { Tezcacoac }\end{array}$ \\
\hline 76 & $\begin{array}{l}\text { Grand calpulli d'Acatl } \\
\text { Yiacapan }\end{array}$ & [Tlaloc] & & & Acatliacapan \\
\hline 77 & Techielli & & & & \\
\hline 78 & Calpulli & & & & \\
\hline
\end{tabular}

FIG. 5. - Les lieux de la liste, les dieux auxquels ils sont dédiés, les moments de leur utilisation et les calpulli desquels ils peuvent être rapprochés.

descriptions et qui donnent cette homogénéité à la liste. Pour comprendre ces liens, il faut se demander comment, et sur quelle base, Sahagún a composé sa liste.

On ne peut rejeter d'emblée l'hypothèse selon laquelle la liste renvoie effectivement à la réalité architecturale du centre cérémoniel. En effet, comme on l'a évoqué plus haut, il est certain que les ensembles dédiés aux dieux principaux tels qu'ils apparaissent dans la liste faisaient partie de l'enceinte cérémonielle. De plus, il n'est pas improbable que l'enceinte ait contenu jusqu'à 80 structures. Cependant, le point épineux de cette hypothèse reste la question des calpulli, car ils sont très souvent présentés par les différents chroniqueurs comme des « églises paroissiales » ou des 
" temples de quartier », ce qui les désigne comme des sanctuaires situés en dehors de l'enceinte et fréquentés par des groupes sociaux déterminés. La remarque de Tezozomoc (1878, p. 228) précisant que, dès leur arrivée à Tenochtitlan, les Aztèques installèrent leur dieu Huitzilopochtli et, autour de lui, les dieux des quinze calpulli, avant de compléter le sanctuaire avec d'autres structures comme un jeu de balle, indique sans doute que des temples dédiés aux dieux des calpulli de Mexico avaient leur place au sein de l'enceinte cérémonielle. Il est donc possible qu'il ne faille pas nécessairement identifier les temples dont la description renvoie à un calpulli à des sanctuaires de quartier ${ }^{88}$ mais plutôt à des édifices « délégués » par les calpulli de la ville à l'intérieur même de l'enceinte.

Une seconde hypothèse, qui n'exclut pas nécessairement la première, me paraît tout à fait plausible : la liste renverrait non pas directement à une réalité architecturale, mais à une image, un "plan » illustrant le Grand Temple. Le faisceau d'indices soutenant cette hypothèse comprend, rappelons-le, d'une part la représentation d'une enceinte conservée dans les Primeros Memoriales (voir Seler 1992, 3, pp. 115-121), et la liste qui l'accompagne et qui s'y rapporte clairement, d'autre part, la mention par Torquemada de l'existence d'une peinture de l'enceinte de Mexico, établie par Sahagún et déjà perdue au début du Xvir ${ }^{\mathrm{e}}$ siècle, et enfin le caractère à la fois précis et inédit des commentaires que livre Sahagún dans sa traduction espagnole, qui ne peuvent provenir d'une observation effective de l'enceinte et qui s'appliquent souvent parfaitement aux représentations indigènes de bâtiments.

En suivant la démarche de Seler (ibid., pp. 115-121) dans son identification des différentes structures du folio des Primeros Memoriales par rapport à la liste ${ }^{89}$, on s'aperçoit que l'informateur anonyme commence par mentionner la pyramide double, qui se trouve dans le centre de l'image, décalée vers le haut, c'est-à-dire vers l'est (voir Figures 6 \& 7). L'énumération se poursuit de proche en proche, en citant successivement les édifices ayant une même orientation, ce qui indique sans doute que la lecture de la carte impliquait une rotation de celle-ci. Cette lecture se termine par les éléments entourant directement la pyramide double et par les ouvertures de l'enceinte. Une approche similaire de la liste du Codex de Florence révèle certains détails très intéressants. On constate ainsi que, comme la liste des Primeros Memoriales, celle du Codex de Florence commence par la pyramide double, dont les temples sont considérés comme des structures indépendantes, et par des édifices de grande importance. Si un plan a bien existé, il est presque certain que ces édifices devaient se situer dans une zone d'importance comparable. De plus, même si les édifices d'un même complexe sont généralement regroupés, on a pu remarquer à plusieurs reprises que certaines descriptions, évoquant indéniablement des édifices d'un même complexe, sont intercalées entre les descriptions d'autres structures, et non énumérées à la suite l'une de l'autre. Les édifices des temples de Yopico, Yiacatecuhtli et Huitzilinquatec ( $n^{\circ} 49$ à 56$)$ en sont un bon exemple. Cette répartition en alternance s'explique difficilement par une énumération orale. En revanche, elle est tout à fait compréhensible dans le cadre d'un commentaire d'image. En effet, dans ce cas, l'informateur, voyageant de proche en proche dans l'image, peut évoquer successivement des édifices très proches à l'intérieur même de l'image, mais dont la proximité ne correspond pas nécessairement à un lien symbolique précis.

L'analyse de la succession des descriptions permet de proposer une répartition des édifices (Figure 8) fondée sur ces observations et sur l'image des Primeros Memoriales. 


\section{EST}

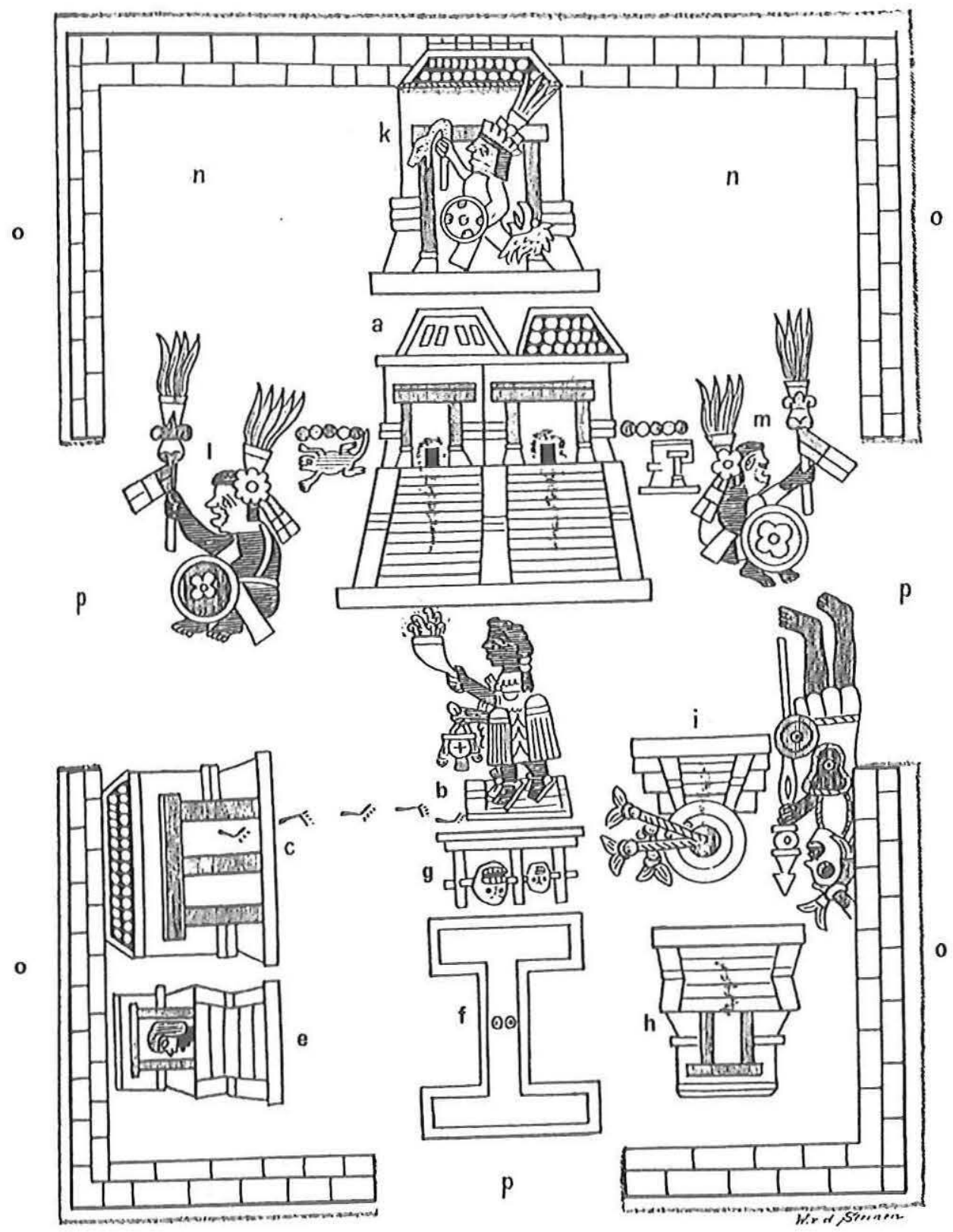

\section{OUEST}

FIG. 6. - Folio 269r des Primeros Memoriales de Sahagún (Seler 1902-1923, 2, p. 771, abb.1). 


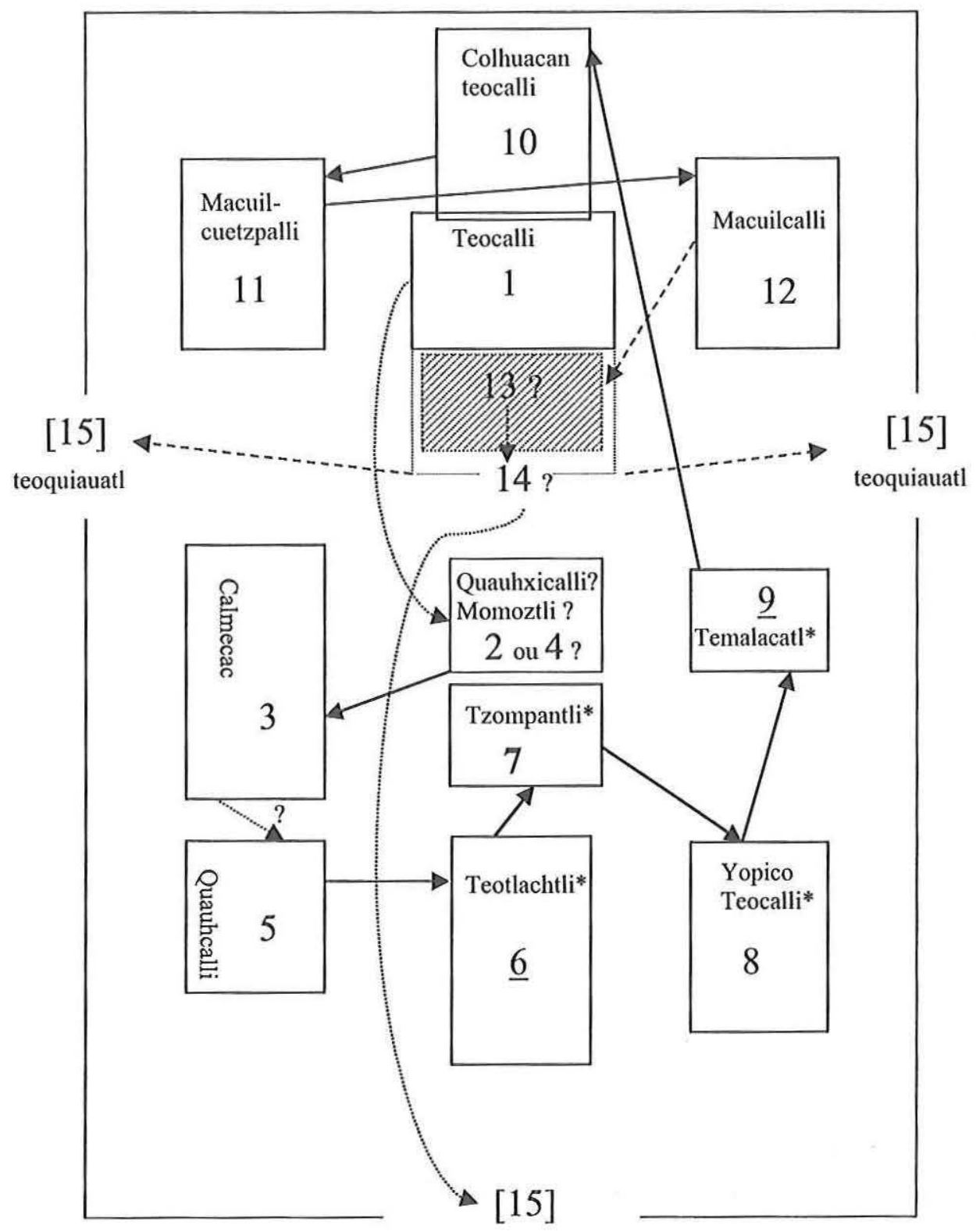

Fig. 7. - Reconstitution du sens de lecture de l'image du Folio 269r des Primeros Memoriales.

Remarques: 1) Les * indiquent que le sommet de l'édifice est dirigé vers le bas de l'image.

2) Un des édifices mentionnés dans la liste n'est pas représenté. D'après Seler, il s'agit du Momoztli ( $\left.n^{\circ} 4\right)$. Il se peut toutefois que ce soit le Quauhxicalli et que le Momoztli soit l'édifice qu'il identifie comme le Quauhxicalli $\left(n^{\circ} 2\right)$. Quoi qu'il en soit, il est clair que cette petite structure est celle qui précède ou qui suit directement le Calmecac $\left(n^{\circ} 3\right)$, car les deux représentations sont reliées par des traces de pas. 


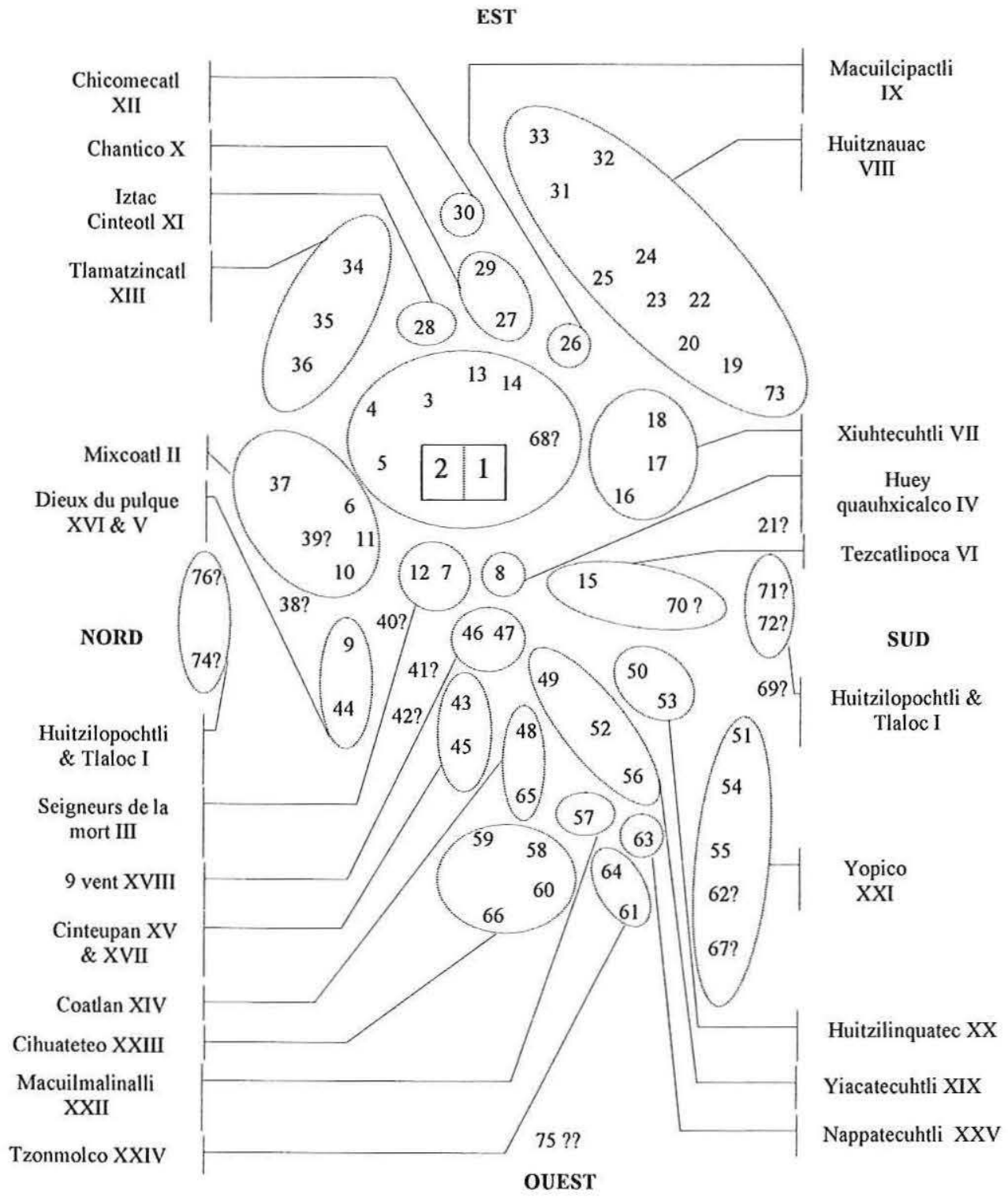

FIG. 8. - Tentative de reconstitution de l'image à laquelle se serait rapportée la liste du Codex de Florence. 
Indépendamment de la stricte localisation des édifices dans le plan, qui restera toujours hypothétique, il apparaît que la liste du Codex de Florence peut être lue de la même manière que la liste et l'image des Primeros Memoriales. Commençant par la pyramide double, qui devait occuper une position centrale, la lecture se poursuit avec des édifices ( $n^{\circ} 6$ à 36 ) qui l'entourent, dont la succession dans la liste indique qu'ils étaient proches les uns des autres. Les édifices n 37 à 41 , qui ont des liens étroits avec le complexe de Mixcoatl et celui de Huitzilopochtli, seraient une charnière dans l'image, car ils marqueraient la fin d'une première boucle autour de la pyramide double, ce qui se traduit par un retour vers les édifices évoqués dans le début de la liste. Il est possible que la troisième série d'édifices ( $n^{\circ} 43$ à 66$)$, probablement composée par une progression de proche en proche au sein de l'image et correspondant peut-être à un changement d'orientation dans le dessin, se termine, elle aussi, par un retour au centre de l'image, avec la source Toxpalatl $\left(n^{\circ} 68\right)$. On observe ensuite que tous les édifices suivants, qui peuvent difficilement être associés à des complexes particuliers, sont qualifiés de «maison » par Sahagún. L'usage de ce terme, que le franciscain utilise pour désigner les petits édifices qui entouraient l'intérieur de l'enceinte, ainsi que la position en fin de liste de ces édifices, qui rappelle que les derniers lieux cités dans la liste des Primeros Memoriales sont l'espace de danse, le mur de serpent et la porte de l'enceinte, suggèrent que la lecture de l'image du Codex de Florence se terminait par le mur d'enceinte, par les édicules qui y étaient greffés et, peut-être, par deux de ses ouvertures.

Il apparaît donc qu'un faisceau d'indices convergents, provenant tant d'informations externes à la liste qu'à sa structuration interne, permet de soutenir l'existence d'une image à laquelle elle se serait rapportée. Malheureusement, dans l'état actuel des sources historiques, la validité de cette hypothèse ne peut être testée davantage. Ces riches et complexes folios du Codex de Florence n'ont donc pas fini de susciter l'intérêt et d'éveiller la curiosité...*

*Manuscrit reçu en janvier 2002, accepté pour publication en mai 2002.

\section{NOTES}

1. Les trois traductions de ce passage du Codex de Florence (ci-après référencé $\mathrm{CF}$ ) actuellement publiées — celle d'Anderson et Dibble, son édition révisée et celle de López Austin (1965) — ont été utilisées dans la préparation de cet article. Cependant, pour des raisons de commodité, et sauf mention contraire, les références qui y sont citées sont celles de la version révisée de la traduction d'Anderson et Dibble.

2. Actuellement, l'appellation " Grand Temple » est souvent réservée à la pyramide double de Huitzilopochtli et Tlaloc, dont les fouilles, commencées à l'occasion du Proyecto Templo Mayor, ont permis de bien en connaître les différentes phases d'agrandissement, à l'exception toutefois de la phase la plus ancienne et de celle que virent les Espagnols.

3. Le titre du chapitre est : «Izcatquj in jmelaoaca, in ixqujch catca inteucal mexica ». Anderson et Dibble le traduisent par "Behold [here] a true [relation] of all [the temples] which were the temples of the Mexicans " (CF 1950-1981, 2, p. 165) et par "Behold here a true [relation] of all the [buildings] which were the Mexicans' temples» (CF 1950-1981, 2, p. 179). López. Austin (1965, p. 76) l'intitule "He aqui la descripción de todos los templos de los mexicanos ».

4. En effet, à ce jour, plus de 30 édifices — grands temples, petits autels et plates-formes — ont été mis au jour dans la zone de l'enceinte cérémonielle (López Luján 2001, p. 715). 
5. Cet article s'appuie sur mon mémoire de DEA, présenté à l'École pratique des hautes études de Paris en 2000 .

6. Cette tendance à l'exagération a notamment été mise en évidence par Seler (1992, 3, p. 117), à propos du nombre de marches que comptait le temple double de Mexico.

7. En réalité, il conviendrait d'orthographier ce terme « $k$ 'u ». Comme il est retranscrit « $c u »$ dans toutes les sources historiques, l'orthographe ancienne a été maintenue tout au long de cet article. Cette remarque orthographique m'a été judicieusement suggérée par un des critiques anonymes qui ont analysé la première version de cette article. Qu'il soit ici remercié pour sa lecture attentive et pour ses remarques, toujours enrichissantes.

8. Au sein de la liste, on remarque que Sahagún qualifie de $c u$ non seulement les édifices que ses informateurs désignent comme des iteupan, des « lieux de dieu » ou des teocalli, des « maisons de dieu », dont on sait, par d'autres sources, qu'ils désignent des temples sur pyramide, mais aussi un certain nombre d'autres édifices (Figure 3). On pourrait avancer l'hypothèse que le terme $c u$ s'applique aux édifices sur pyramide, si Sahagún ne donnait pas d'autres détails qui ruinent définitivement cette suggestion. Ainsi, lorsqu'il évoque un " petit cu rond, d'environ 3 brasses de large et 1,5 de haut, sans aucune couverture " (Quauhxicalco ${ }^{\circ} 15$ ), un « cl ou momoztli » (Autre Quauhxicalco ${ }^{\circ} 16$ ), un « cu bas, carré, avec des marches sur les quatre faces » (Tochinco $n^{\circ}$ 9) ou encore, lorsqu'il qualifie de $c u$ un brasier qu'il décrit par ailleurs comme un grand autel avec des marches sur quatre côtés sur lequel était allumé le brasier (Sahagún 1999, p. 137), on ne peut que constater la flexibilité de ce terme.

9. Le Tzompantli de Mixcoatl $\left(\mathrm{n}^{\circ} 6\right)$, le Grand tzompantli $\left(\mathrm{n}^{\circ} 41\right)$, le Tzompantli de Yopico $\left(\mathrm{n}^{\circ} 55\right)$ et trois autres structures simplement appelées tzompantli $\left(\mathrm{n}^{\circ} 18,33,56\right)$.

10. Le terme "pelote " est habituellement et improprement utilisé par les chroniqueurs du Xvi" siècle pour désigner la balle de ulli qu'utilisaient les Indiens dans leurs jeux de balle (Taladoire 1981, p. 33). Dans cet article, cette ancienne appellation n'a été conservée que dans les citations.

11. Cette identification pose malgré tout un problème, car la liste ne présente que trois tlacochcalco alors que l'enceinte était sans doute ouverte sur ses quatre côtés, comme l'aftirirme Durán(1984, 1, p. 22). Même si on ne peut pleinement expliquer cette discordance, il faut tenir compte du fait que l'ouverture qui donnait sur la chaussée menant à la lagune ne nécessitait peut-être pas une défense aussi importante que les autres et que, de toute manière, il ne peut pas être démontré que la liste de Sahagún soit parfaitement exhaustive.

12. Au moins un point d'eau aménagé a été mis au jour dans le site du Grand Temple de Tlatelolco, la ville jumelle de Tenochtitlan, située dans la partie nord de l'ile de Mexico et dont on sait qu'elle possédait une enceinte cérémonielle à l'image de celle de Tenochtitlan. Cet édifice, qualifié de "puits sacré », est décrit comme un petit bassin, entouré d'un ensemble de marches qui descendent jusqu'au centre du puits (González Rul 1998, p. 98).

13. Un second édifice est appelé Netlatiloyan ( $\left.\mathrm{n}^{\circ} 38\right)$. Cette appellation dérive du verbe tlatia, qui signifie " cacher ", mais aussi " brûler ". La description de l'édifice n ${ }^{\circ} 59$ permet de lever cette ambiguitté, car les informateurs de Sahagún précisent que des peaux de victimes y étaient déposées. Il s'agit donc sans doute d'une cache cérémonielle, semblable à celles dans lesquelles étaient enterrées les peaux des personnificateurs de Xipe, à la fin de Tlacaxipehualiztli. Quant à l'édifice $\mathrm{n}^{\circ} 38$, il est très probable, comme le suggère López Austin (1965, p. 89), qu'il s'agit d'un brasier, en raison du lien étroit existant entre le sacrifice par le feu et Nanahuatl, dont un personnificateur mourait à cet endroit.

14. Plusieurs auteurs avancent cette hypothèse, notamment Nicholson (Sahagún 1997, p. 117) et Pasztory (1998, p. 110).

15. Le folio de ce codex, élaboré suite à son séjour à Tepepulco, représente une enceinte cérémonielle dans laquelle figurent huit édifices et quatre personnages. Une liste non commentée accompagne cette image qui a été interprétée, à la suite de Seler (1992, 3, pp. 115 et ss.), comme un plan du centre cérémoniel de Mexico. Quoi qu'il en soit de l'identification toujours débattue de ce lieu, cette page est extrêmement importante, car elle démontre que Sahagún a au moins une fois récolté des informations tant figurées qu'écrites au sujet d'une même enceinte cérémonielle. Pour une analyse détaillée de cette planche des Primeros Memoriales, voir les commentaires de Nicholson dans la traduction de Sullivan (Sahagún 1997, pp. 117-119).

16. Cette description pouvait probablement aussi s'appliquer à des petites structures, comme celle qui est actuellement qualifiée d'« autel tzompantli » et qui a été retrouvée près de la pyramide principale. Cependant, la multiplication des détails se rapportant à l'aspect extérieur d'édifices théoriquement détruits ne s'explique que par la lecture d'une image. Ainsi, comment Sahagún saurait-il que le Coacalco $\left(n^{\circ} 14\right)$ est une " salle grillagée comme une prison » et que le lieu Ilhuicatitlan $\left(n^{\circ} 40\right)$ est une " grosse et haute colonne ", alors que ses informateurs ne donnent aucun élément permettant de le déduire et que ces lieux ne sont pas connus par ailleurs? 
17. La thèse de doctorat de Michel Graulich est, jusqu'à présent, toujours la seule étude qui envisage les rites aztèques de manière systématique. Comme ceux-ci avaient en grande partie lieu dans l'enceinte cérémonielle de Mexico, cet ouvrage s'est révélé particulièrement utile pour l'étude de cette liste d'édifices religieux. Il en existe à présent une traduction espagnole (Graulich, 1999, Ritos aztecas. Las fiestas de las veintenas, México, Instituto Nacional Indigenista).

18. González Torres (1985, pp. 161-171) évoque ces références aux calpulli dans son chapitre traitant des lieux de sacrifices.

19. J'entends ici par " ensemble » ou « complexe », un temple et les différentes structures qui lui sont exclusivement associées. Ces annexes sont détaillées notamment par Durán (escalier, patio, calmecac, etc.). Il faut sans doute leur ajouter le tzompantli et quelques annexes spécifiques, comme le brasier pour le temple de Xiuhtecuhtli.

20. Pour une meilleure compréhension des rapprochements proposés ci-dessous, il est utile de consulter régulièrement la liste du Codex de Florence ainsi que la Figure 4.

21. L'un d'eux, Macuilcalli, est présenté ailleurs comme un des dieux des lapidaires (CF 1950-1981, 9, pp. 79-80).

22. Ou qu'ils en étaient proches.

23. Appelés Totec tlamacazqui et Tlalocan tlamacazqui (Sahagún 1999, p. 214).

24. Pour désigner les personnes qui résidaient dans ce calmecac, les informateurs de Sahagún utilisent le terme "tlamaceuhque ", qui signifie littéralement "pénitent " (Molina 1944, p. 125), "dévot, religieux » (Siméon 1963, p. 548), ce que rendent fidèlement les traductions d'Anderson et Dibble (édition révisée, p. 182) et de López Austin (1965, p. 82). Cependant, on remarquera que Sahagún (1999, p. 159) et les mêmes Anderson et Dibble (CF 1950-1981, $1^{\mathrm{e}}$ édition, p. 168) ont choisi de le traduire par " prêtre ». Ce choix est tout à fait justifié, parce que, dans le Codex de Florence, hormis un passage où il désigne les dieux Nanahuatzin et Tecuciztecatl (CF 1950-1981, 7, p. 5), le terme "tlamaceuhque " est exclusivement utilisé pour qualifier les prêtres (tlamacazque) (voir par exemple CF 1950-1981, 6, pp. 114 et 209).

25. La coutume de s'emparer des images divines des vaincus est attestée par d'autres sources, mais leur endroit de conservation n'y est pas évoqué. On sait cependant qu'une salle du palais de Montezuma, appelée Coacalli, était destinée à héberger les visiteurs étrangers de passage, alliés ou ennemis (CF 1950-1981, 8, p. 44). Il semble donc qu'à l'image de la résidence impériale, le centre cérémoniel possédait un lieu de résidence spécifique pour les « hôtes » divins étrangers.

26. Ce rapprochement concorde avec les indications des chroniqueurs, mais il est contesté par les données archéologiques, car la structure identifiée comme le Grand Tzompantli ne se situe pas dans les environs immédiats de la pyramide principale (Vega Sosa 1979, p. 70).

27. Les termes de la description de l'Itepeioc font directement allusion au Temple de Huitzilopochtli, car ils le présentent comme le lieu de " naissance " (de tlacati, naître) de Huitzilopochtli qui, d'après les mythes, a eu lieu sur le Coatepec. Or le Temple de Huitzilopochtli et la grande pyramide sur laquelle il repose sont précisément identifiés à cette montagne, comme en témoigne notamment la statue de la Coyolxauhqui démembrée qui gît au bas de la pyramide.

28. Apparemment, les opérations de fabrication avaient lieu dans deux endroits distincts, car le premier édifice est décrit comme le lieu de cuisson de la pâte et le second comme le lieu de sa mise en forme.

29. D'après Seler (1992, 3, p. 115), cette placette correspond à la place appelée Empedradillo au début du $\mathrm{xx}^{\mathrm{e}}$ siècle, situće du côté ouest de la cathédrale.

30. Celui de la naissance du Soleil et de la Lune à Teotihuacan (cf. infra, le Netlatiloyan n ${ }^{\circ} 38$ ), celui de la victoire de Quetzalcoatl au Mixcoatepec et de celle de Huitzilopochtli au Coatepec (voir Graulich 1980, pp. 499 et ss.).

31. L'épouse traditionnelle du Seigneur de la Mort est Mictlancihuatl, la Dame de la Mort, mais un certain nombre de vieilles déesses, et notamment Cihuacoatl, peuvent se substituer à elle (voir Graulich 1980, 2, pp. 548-549).

32. En effet, la courte description du Codex de Florence est le parfait résumé de celle de Durán. Les éléments spécifiques qu'elles ont en commun, comme le jour du déroulement du sacrifice (4 Mouvement), le fait que le sacrifice avait lieu à midi, et la personnalité du dieu auquel ces rites sont dédiés, permettent de supposer qu'il s'agit de la même fête. Carlos Javier González, qui a lu mon mémoire de DEA après sa soutenance et qui, ensuite, a eu la gentillesse de me faire des commentaires tout à fait utiles pour la suite de ma recherche, a attiré mon attention sur le fait que Durán se réfère à plusieurs occasions au patio contenant les pierres appelées Temalacatl et Quauhxicalli comme " patio cuauhxicalco », et qu'il décrit toujours ce dernier en étroite association avec le Cuacuauhtinchan, le « temple du soleil », ce qui renforce l'identification du Huey Quauhxicalco qu'évoquent les informateurs de Sahagún au « temple du soleil » que présente Durán. 
33. Les liens entre les dieux de l'ivresse et du pulque et le lapin sont bien connus. Voir par exemple les Costumbres f. $354 \mathrm{r}$ et ss.

34. Le texte nahuatl parle d'jxiptla tochinco, de "personnificateur de Tochinco ». Or le suffixe co est un locatif. Tochinco ne donc peut pas être le nom d'une divinité, en théorie.

35. Tepuztecatl signifie en effet le « celui de Tepuztlan » (Garibay 1999, p. 947).

36. Les informateurs de Sahagún avancent cette localisation temporelle avec quelque réserve, puisqu'ils indiquent que les sacrifices avaient lieu " peut-être lorsque régnait le signe ce miquiztli » (CF 1950-1981, 2, p. 192). Sahagún ne reprend pas cette nuance dans son commentaire.

37. Comme l'attestent le Codex Magliabechiano (f. 39) et le Codex Telleriano-Remensis (f. 163-164).

38. L'érection de cet arbre est bien connue, car elle est un des rites centraux de la vingtaine de Xocotl Huetzi, également dédiée à Xiuhtecuhtli.

39. On y apprend, en effet, qu'une fois les victimes jetées dans le brasier appelé Teccalco, un personnage écureuil venait danser à cet endroit (CF 1950-1981, 2, p. 129).

40. López Austin (1965, p. 83) considère que la description renvoie non pas à Teotl Eco mais à Xocotl Huetzi.

41. Voir aussi Durán $(1984,1$, p. 120).

42. Il est ainsi cité dans une liste de temples (CF 1950-1981, 9, p. 37) comprenant également le Yopico, le Pochtlan et le Tlamatzinco, trois lieux qui figurent dans la liste des édifices du Grand Temple du Codex de Florence, mais qui sont également des noms de calpulli de Mexico. Ailleurs dans le même codex (ibid., 2, p. 71), il est présenté comme le lieu où était façonnée l'efligie de Huitzilopochtli, lors de la fète de Toxcatl. Il n'est pas toujours possible de préciser s'il s'agit du même temple que celui qui est évoqué dans la liste ou bien d'un temple situé dans le quartier de Huitznauac.

43. Ainsi, d'après la liste, les victimes qui y sont mises à mort représentent les ennemis mythiques du dieu et, ailleurs dans le Codex de Florence (1950-1981, 2, p. 71), il est précisé que c'est à cet endroit qu'était façonnée l'image du dieu pour la fète de Toxcatl.

44. Il est ainsi décrit comme le « compagnon » (Sahagún 1999, p. 164) ou le « frère » de Huitzilopochtli (Torquemada 1969, 2, p. 155). En fait, ce dieu apparaît dans les deux vingtaines au cours desquelles Huitzilopochtli et Tezcatlipoca sont conjointement honorés, Toxcatl et Panquetzaliztli, et semble, dans les deux cas, avoir une position de contrepoids par rapport au dieu principal de la vingtaine : en Toxcatl, il se présente comme Huitzilopochtli, et en Panquetzaliztli, il incarne Tezcatlipoca, aux côtés de Huitzilopochtli (Graulich 1980, p. 665).

45. Le nom du prêtre chargé de superviser le calmecac de Huitznauac, Huitznaua teuatzin Omacatl (CF 1950-1981, 2, p. 206), indique également un rapport étroit entre Omacatl et le calpulli de Huitznauac.

46. Le « lieu du jeu de balle du miroir », de " tezcatl » qui signifie miroir et "tlachtli » qui désigne le terrain de jeu de balle.

47. En effet, parmi les nombreux exemples de caches mis au jour dans l'enceinte, certaines contenaient des épines d'agaves (Matos Moctezuma et al. 1998, p. 18). Pour une étude approfondie des caches cérémonielles découvertes dans le Grand Temple de Mexico, voir les travaux de López Luján, notamment celui de 1993. Pour les caches de Tlatelolco, voir notamment Guilliem Arroyo (1996).

48. Huitznauac signifie " près des épines » et Huitztepehualco « où les épines sont répandues » (Garibay 1999, p. 930).

49. Le dictionnaire de Molina (1944) indique que ce terme signifie « avoir des crouttes ou la gale ».

50. Comme en témoignent notamment la peinture représentant Cinteotl dans le temple de Tlaloc de la phase II du Templo Mayor, et les attributs de Xipe que revêt Cinteotl-Xochipilli dans l'illustration de la vingtaine de Huey Tecuilhuitl du Codex Borbonicus (f. 27), par exemple. Voir aussi Graulich (1980, p. 700).

51. Pour une analyse des événements mythiques célébrés en I Fleur, voir, entre autres, Graulich (1980, pp. 46-47).

52. Le domaine de cette divinité transparaît notamment dans son nom. Garibay (1999, p. 927) le traduit par « dans le foyer », et Seler (1992, 2, p. 265) par « dans la maison ».

53. López Austin (1969, p. 86) considère qu'il s'agit de la contraction de « Chicome Ehecatl », « 7 vent ».

54. Graulich (1980, pp. 700-701) considère que la divinité ici évoquée pourrait être Chicomecoatl et non Chicomecatl, en raison du fait qu'en plusieurs circonstances des divinités du maïs (Xochipilli, 5 Fleur, Cinteotl ou Xilonen) sont fètées en même temps que Chantico-Cihuacoatl, dans le cadre de fêtes mobiles. Or les descriptions qui précèdent celle du temple de Chicomecatl $\left(\mathrm{n}^{\circ} 30\right)$ se rapportent, elles aussi, à la fête mobile 1 Fleur et évoquent précisément les dieux Iztac Cinteotl, 5 Caïman et Chantico.

55. Cette indication, inédite dans le Codex de Florence, est confirmée par la description d'Ochpaniztli par Durán (1984, 1, pp. 137-140). 
56. " iteupan chicomecoatl».

57. Cette vingtaine est également appelée Xilomaniztli, notamment dans la description des édifices dont il est question ici.

58. Pour les apparitions de « 9 Vent » dans les sources, voir Graulich (1994-1995, p. 34).

59. Les liens qui unissent Yiacatecuhtli, le calpulli de Pochtlan et les marchands sont assez bien connus, sans doute parce que Sahagún consacre presque un livre entier de son Historia (le livre 9) à cette profession et à ses usages. Pour mettre en lumière ces liens, citons, par exemple, le nom du prêtre de Yiacatecuhtli qui est, d'après la liste des prêtres, Pochtlan Teuhua Yiacatecuhtli, mais aussi le fait que c'est dans ce calpulli, ou dans celui d'Acxotlan, que les esclaves offerts par les marchands en Panquetzaliztli veillaient après avoir bu du pulque au Temple de Huitzilopochtli (CF 1950-1981, 9, p. 63). Le nom nahuatl pour désigner les marchands est, à lui seul, éclairant puisqu'ils sont appelés pochteca.

60. On le retrouve notamment dans le Codex de Florence (1950-1981, 2, p. 45), où il est qualifié de "Totec iteupan ». Le prêtre de Xipe est appelé "Xippe Iopico Teuhua » dans la liste des prêtres (ibid., p. 213).

61. Les informateurs de Sahagún indiquent que son personnificateur était orné d'ocre rouge, de plumes de dinde blanche et d'une " cape de Totec " (CF 1950-1981, 2, p. 213), attributs qui s'apparentent peut-être aux ornements que les capteurs revêtent pendant la préparation de la fête, en Quauitl Eua (ibid., pp. 45-46).

62. Il faut noter que, contrairement aux autres descriptions de calmecac, celui-ci n'est pas présenté explicitement comme un lieu de résidence de prêtres.

63. À propos du lien entre Xipe et l'Anahuac, voir Seler (1992, 2, p. 245). Quant aux hôtes hébergés à cet endroit, il est possible qu'il s'agisse des seigneurs étrangers invités à Mexico pour assister aux sacrifices de Tlacaxipehualiztli (CF 1950-1981, 2, p. 55).

64. Les noms de ces dieux signifient respectivement « 5 Herbe » et « Celui qui s'est fait homme pour notre cause " (López Austin 1965, p. 94).

65. Comme l'a montré Seler (1992, 2, p. 259), les dieux « 5 » ont « un air de famille [et] ils semblent faire office de compagnons et de frères de Macuilxochitl [-Xochipilli] ".

66. Ce terme désigne les femmes mortes en couches, qui étaient divinisées car leur mort et celle de leur enfant étaient assimilées à la mort glorieuse du guerrier combattant (la parturiente) ayant capturé un prisonnier (l'enfant). Elles jouissaient d'un devenir post-mortem particulier et leurs apparitions nocturnes étaient très redoutées (Sahagún 1999, p. 95).

67. Un personnage dans le même accoutrement est représenté dans l'image d'Ochpaniztli du Codex Borbonicus (f. 29).

68. Le sacrifice des déesses de l'eau en Ochpaniztli est évoqué par Durán (1984, 1, pp. 136-137) et représenté dans le Codex Borbonicus (f. 29).

69. Atlauhco est aussi traduit par «lieu du précipice » (Garibay 1999, p. 918 ; Graulich 1980, p. 381).

70. Selon les auteurs, au sommet du temple (CF 1950-1981, 2, p. 58) ou au pied des escaliers (Durán 1984, 1, p. 102).

71. En tant que femme sacrifiée, la personnificatrice d'Atlatonan peut être considérée comme une des Cihuateteo, une « femme divine ». Par ce détail, on remarque la cohérence de la liste des édifices, puisque ces indications concordent avec la description de la cache Netlatiloyan ( $\left.n^{\circ} 59\right)$. Les informateurs de Sahagún ne précisent pas le sexe des personnes incarnant les dieux du maïs, sacrifiées en même temps qu'Atlatonan. Dans le cas où ce seraient des femmes - ce qui est probable puisque Sahagún (1999, p. 160) évoque ailleurs une personnificatrice sacrifiée dans le temple du Cinteotl blanc et que Cinteotl est tantôt incarné par un homme (CF 1950-1981, 2, p. 120), tantôt par une femme (Costumbres 1945, p. 41) -, elles entreraient également dans la catégorie des Cihuateteo.

72. Ils personnifient respectivement le Xiuhtecuhtli vert, le jaune, le blanc et le rouge.

73. López Austin (1965, p. 97) traduit ces noms respectivement par " Ceux du passage des plumes » et "Ceux de la colonne de pierre ».

74. Cihuatontli se traduit par "petite femme » et Nancotlaceuhqui par «celle qui apaise en répondant (?) " (López Austin 1965, p. 97). Selon Sahagún, leur sacrifice avait lieu sur une petite plate-forme, au pied du Tzonmolco, et non sur le temple proprement dit.

75. La liste des prêtres (CF 1950-1981,2, p. 211) indique qu'on y conservait le bois destiné à être brûlé en honneur de Ixcoçauhqui, un autre nom du dieu du feu.

76. Il est également considéré comme un des dieux du pulque, puisque son prêtre s'appelle « Ome Tochtli Nappatecuhtli » (CF 1950-1981, 2, p. 210). Ces dieux étaient également vénérés en Tepeilhuitl, par des sacrifices de victimes les incarnant (voir Graulich 1980, p. 465).

77. Voir note 13. 
78. Voir note 11.

79. Le Codex Magliabecchiano précise ainsi qu'un des temples de Tezcatlipoca portait le nom de tlacochcalcatl. En Toxcatl, le petit temple au sommet duquel est sacrifié le personnificateur de Tezcatlipoca est appelé tlacochcalco (CF 1950-1981, 2, p. 71). De plus, selon Seler (1992, 2, p. 262), le dieu Tlacochcalco Yaotl, le "guerrier de la maison des javelines " n'est autre que Tezcatlipoca. À ce sujet, voir Olivier (1997, pp. 193-195).

80. Le fait que ce lieu soit qualifié de quauhquiauac, " de la porte des aigles », confirme qu'il s'agit avant tout d'un arsenal associé à une entrée de l'enceinte. En effet, un lieu appelé quauhquiauac est cité à deux reprises dans le Codex de Florence (CF 1950-1981, 2, p. 71 ; Sahagún 1999, 12, p. 746). Dans les deux cas, ce lieu est évoqué au moment oủ les protagonistes entrent dans l'enceinte cérémonielle, avant d'atteindre la cour et le temple de Huitzilopochtli.

81. Tezcacoac peut se traduire par le « lieu du serpent de miroir » (López Austin 1965, p. 100). Selon Durán (1984, 1, p. 154), deux jeunes filles étaient choisies parmi les descendants d'un grand prince du nom de Tezcacoac pour être sacrifiées, en Teotl Eco, sur une pierre cuauxcicalli.

82. Ce terme est traduit par Anderson et Dibble comme des « bannières de papier humaines », tandis que López Austin en donne la traduction littérale, « poussières humaines ».

83. Voir plus haut, notre commentaire à propos du Iztac Cinteotl Iteupan $\left(\mathrm{n}^{\circ} 28\right)$.

84. Selon le Codex de Florence (1950-1981, 2, p. 208), le prêtre d'Atempan est chargé des préparatifs du sacrifice de Toci en Ochpaniztli. À plusieurs reprises, un lieu appelé Atempan est qualifié de « maison » de Toci. En Ochpaniztli, c'est là que les Huastèques chargés d'accompagner la personnificatrice de la déesse font pénitence (ibid., p. 208) et que le second personnificateur de la déesse se retire, une fois que le masque de Cinteotl a été laissé à Popotl Temi (ibid., p. 122).

85. Sahagún interprète cette indication comme évoquant des petites structures construites contre l'intérieur du mur d'enceinte. Un des critiques anonymes ayant relu la première version de cet article - que je remercie d'ailleurs grandement pour toutes ses précisions et pour ses remarques judicieuses et instructives m'indique que Matos Moctezuma considère, lui, qu'il pourrait s'agir des petits édicules découverts à l'extrémité orientale de l'enceinte cérémonielle, sur la plate-forme qui borde celle-ci. La question de l'identification des édifices auxquels font allusion les informateurs de Sahagún dans ce passage reste très problématique, en raison du fait que ce passage est particulièrement bref et peu explicite, et que les autres mentions évoquant des édifices appelés calpulli sont laconiques et contradictoires. Par ailleurs, l'identification des édifices mis au jour par l'archéologie est rendue diflicile par la disparition presque totale de la dernière phase de construction de l'enceinte cérémonielle de Mexico, celle que virent et détruisirent les Espagnols à leur arrivée et qui est sans doute celle à laquelle se réfère la liste du Codex de Florence. Pour ces raisons, l'hypothèse de Matos Moctezuma, bien qu'intéressante, ne peut pas être testée davantage.

86. Je tiens également à remercier ce même lecteur anonyme de m'avoir indiqué que Sanders a utilisé cette indication du Codex de Florence pour proposer une interprétation des quinze structures pyramidales construites sur la plate-forme qui borde la Ciudadela de Teotihuacan. Considérant, à juste titre me semble-t-il, que les petits édifices appelés calpulli dépendaient sans doute des différents quartiers de la ville, cet auteur suggère que les constructions entourant la Ciudadela ont pu être des "ambassades" ou des "représentations » des temples des calpulli. Cette théorie est peut-être à rapprocher de celle qui est généralement avancée pour interpréter les pyramides à rampes de Pachacamac (Pérou), dont on dit souvent qu'elles sont des " ambassades » de temples régionaux au sein du centre cérémoniel de Pachacamac (pour une critique de cette théorie, voir notamment Peter Eeckhout, 1999, Pachacamac durant l'intermédiaire récent. Étude d'un site monumental préhispanique de la côte centrale du Pérou, BAR, International Series, 747, Oxford, pp. 405-407 et chapitre 18). Dans le cas du site péruvien comme dans celui de Teotihuacan, ces interprétations ne peuvent être vérifiées.

87. Si on accepte l'identification de Chantico comme étant la patronne du calpulli de Cihuatecpan (Van Zantwijk 1966, pp. 181-183), on retrouve effectivement les divinités des quinze calpulli les plus anciens au sein de la liste : Xipe Totec (Yopico), Tezcatlipoca (Tlacochcalco); Omacatl-Tezcatlipoca-Tlacahuepan Cuexocotzin (Huitznauac) ; Huitzilopochtli (Tlacatecpan) ; Ixcozauhqui-Xiuhtecuhtli (Tzonmolco) ; Toci (Atempan), Quetzalcoatl ou Ayopechtli (Texcacoac) ; Tlamatzincatl (Tlamatzinco) ; Chiconau ehecatl (Mollocotitlan et Chililico-Nonohualco); Chantico (Cihuatecpan); Izquitecatl, qui est, d'après Sahagún (1999, p. 51), un « frère de Ometochtli » (Izquitlan); Iztac Cinteotl ou Tlatauhqui Cinteotl ou Atlatonan (Milnahuac); Omacatl, Yiacatzintli et Coatlxoxouqui (Coaxoxoucan); les Cihuateteo et Chicomecoatl (Aticpan). Pour plus de détails, voir Van Zantwijk (1965, 1966 et 1985). González Torres (1985, p. 162, cuadro 3) reprend une liste de calpulli légèrement différente de celle de Van Zantwijk, mais parvient à des rapprochements très comparables. 
88. L'existence de ces temples distribués dans les différents quartiers de la ville est attestée par de nombreux chroniqueurs, qui les qualifient généralement d'" églises de quartier " (Sahagún 1999, p. 164).

89. Certaines réserves doivent être formulées, il me semble, quant à son identification du Coatenamitl, en raison de la découverte plus récente d'un « mur de serpent » qui entoure non pas l'ensemble de l'enceinte sacrée, mais la plate-forme de la pyramide principale.

\section{RÉFÉRENCES BIBLIOGRAPHIQUES}

Liste des abréviations :

INAH Instituto Nacional de Antropología e Historia.

SEP Secretaria de Educación Pública.

UNAM Universidad Nacional Autónoma de México.

BARrera Rivera, José Alvaro, 1999. - « El rescate arqueológico en la Catedral y en el Sagrario metropolitanos de la Ciudad de México ", in : Eduardo Matos Moctezuma (coord.), Excavaciones en la Catedral y Sagrario metropolitanos, INAH, México, pp. 21-49.

Boone, Elizabeth H. (ed.), 1987. - The Aztec Templo Mayor : a symposium at Dumbarton Oaks, 8th and 9th October 1983, Dumbarton Oaks Research Library and Collection, Washington.

BrodA, Johanna, 1971. - « Las fiestas aztecas de los dioses de la lluvia », Revista española de antropología americana, 6, pp. 245-327, Madrid.

Broda, Johanna, David Carrasco \& Eduardo Matos Moctezuma, 1999. - The Great Temple of Tenochtitlan. Center and Periphery in the Aztec World, University of California Press, Berkeley-Los Angeles-London.

Carrasco, Pedro, 1971. - « Social Organization of Ancient Mexico », in : Robert Wauchope (ed.), Handbook of Middle American Indians, vol. 10, University of Texas Press, Austin, pp. 349-375.

CAso, Alfonso, 1956. - Los barrios antiguos de Tenochtitlán y Tlatelolco, Memorias de la Academia Mexicana de la Historia, 15, México.

—, 1967. - Los calendarios prehispánicos, UNAM, México.

Codex Magliabechiano and the lost prototype of the Magliabechiano group, 1983. - E. Boone (ed.), University of California Press, Berkeley.

Codex Telleriano-Remensis. Ritual, Divination, and History in a Pictorial Aztec Manuscript, 1995. - E. Quiñones Keber (ed.), University of Texas Press, Austin.

Códice Chimalpopoca : Anales de Cuauhtitlan y Leyenda de los Soles, 1945. - P. F. Velázquez (trad.), Imprenta Universitaria, México.

Contés, Hernán, 1996. - La conquête du Mexique, D. Charnay (trad.), La Découverte, Paris.

Costumbres, fiestas, enterramientos y diversas formas de proceder de los Indios de Nueva España, 1945. - Tlalocan, 2 (1), pp. 37-63.

Couvreur, Aurélie, 1999-2000. — Le Grand Temple de Mexico selon Fray Bernardino de Sahagún, mémoire de DEA, 2 vol., École pratique des hautes études, Paris.

Durán, (Fray) Diego, 1984. - Historia de las indias de Nueva España e islas de tierra firme, 2 vol., Editorial Porrúa, México.

GARIBAY, Angel María,1999. - « Vocabulario de las palabras y frases en lengua nahuatl que Sahagún usa en su obra », in : Historia general..., Sahagún, 1999, pp. 913-963. 
GonzÁlez Rul, Francisco, 1998. - Urbanismo y arquitectura en Tlatelolco, inAH, México.

GONZÁlez TORres, Yolotl, 1985. - El sacrificio humano entre los mexicas, SEP, México.

Graulich, Michel, 1980. - Mythes et rites du Mexique central préhispanique, Thèse de doctorat, 3 vol., Université Libre de Bruxelles.

—, 1993-1994. — « Fêtes mobiles et occasionnelles des Aztèques », Annuaire de la section des Sciences religieuses, 102, pp. 27-31, École pratique des hautes études, Paris.

—, 1994-1995. — « Fêtes mobiles et occasionnelles des Aztèques (suite et fin) », Annuaire de la section des Sciences religieuses, 103, pp. 33-39, École pratique des hautes études, Paris.

Gresle-Pouligny, Dominique, 1999. - Un plan pour Mexico. Les représentations de la cité et l'imaginaire européen ( $\mathrm{XvI}^{\mathrm{e}}-\mathrm{xvIII}^{\mathrm{e}}$ siècles), L'Harmattan, Paris-Montréal.

Gulluiem Arroyo, Salvador, 1999. - Ofrendas a Ehecatl-Quetzalcoatl en México-Tlatelolco. Proyecto Tlatelolco, 1987-1996, Colección científica, Serie Arqueología, INAH, México.

López Austin, Alfredo, 1965. - «El Templo Mayor de México-Tenochtitlan según los informantes indígenas ", Estudios de Cultura Nahuatl, 5, pp. 75-102.

López Luján, Leonardo, 1993. - Las ofrendas del Templo Mayor de Tenochtitlan, inAH, México.

—, 2001. — «enochtitlán : Ceremonial Centers », in : Susan T. Evans \& David L. Webster (eds.), Archaeology of Ancient Mexico and Central America : an Encyclopedia, Garland Publishing, New York, pp. 712-717.

Matos Moctezuma, Eduardo, 1984. — « Los edificios aledaños al Templo Mayor », Estudios de Cultura Nahuatl, 17, pp. 15-21.

—, 1999. — « Sahagún y el recinto ceremonial de Tenochtitlan », Arqueología Mexicana, 6 (36), pp. 22-29.

Matos Moстеzuma, Eduardo (coord.), 1979. - Trabajos arqueológicos en el centro de la Ciudad de México (Antología), INAH, SEP, México.

—, 1999. - Excavaciones en la Catedral y el Sagrario metropolitanos. Programma de arqueología urbana, INAH, México.

Matos Moctezuma, Eduardo, Francisco Hinojosa \& José Alvaro Barrera, 1998. " Excavaciones arqueológicas en la catedral de México », Arqueología Mexicana, 6 (31), pp. 12-19.

Molina, Alonso de (Fray), 1944. - Vocabulario en lengua castellana y mexicana, Ediciones cultura hispánica, Madrid.

Monzón, Arturo, 1949. - El calpulli en la organización social de los tenochca, unam, México.

Motolinía, (Fray) Toribio de Benavente, 1995. - Historia de los indios de Nueva España : relación de los ritos antiguos, idolatrías y sacrificios de los Indios de Nueva España, y de la maravillosa conversión que Dios en ellos ha obrado, $6^{\mathrm{e}}$ éd., Editorial Porrúa, México.

Olivier, Guilhem, 1997. - Moqueries et métamorphoses d'un dieu aztèque. Tezcatlipoca, le "Seigneur au miroir fumant », Musée National d'Histoire Naturelle, Mémoires de l'Institut d'Ethnologie, Paris.

Oviedo y Valdés, Gonzalo Fernández de, 1945. - Historia general y natural de las Indias, islas y tierra firme del mar oceano, 14 vol., Editorial Guarania, Asunción.

PASZtory, Esther, 1998. - Aztec Art, $2^{\mathrm{e}}$ éd., University of Oklahoma Press, Norman.

SaHaGún, (Fray) Bernardino de, 1950-1981. - Florentine Codex, General History of the Things of New-Spain, A. J. O. Anderson \& C. E. Dibble (trads.), 13 vol., The School of American Research, University of Utah, Santa Fe. 
—, 1980. - Códice de Florencia, fac-similé, 3 vol., Archivo General de la Nación, México.

—, 1993. - Primeros Memoriales, fac-similé, University of Oklahoma Press, Norman.

—, 1997. - Primeros Memoriales, T. D. Sullivan (trad.), University of Oklahoma Press, Norman.

-, 1999. - Historia general de las cosas de Nueva España, escrita por Fr. Bernardino de Sahagún, Angel María Garibay (comm.), $10^{\mathrm{e}}$ ed, Editorial Porrúa, México.

SELER, Eduard, 1902-1923. - Gesammelte Abhandlungen zur Amerikanischen Sprach- und Altertumskunde, 5 vol., Asher und Co., Berlin.

-, 1963. - Comentarios al Códice Borgia, 3 vol., Fondo de Cultura Económica, México.

—, 1992. - Collected Works in Mesoamerican Linguistics and Archaeology, 5 vol., Labyrinthos, Culver City.

Siméon, Rémi, 1963. - Dictionnaire de la langue nahuatl ou mexicaine, Akademische Druck-u. Verlagsanstalt, Graz.

TAladoire, Eric, 1981. - Les terrains de jeu de balle (Mésoamérique et Sud-Ouest des ÉtatsUnis), Mission Archéologique et Ethnologique Française au Mexique, Mexico.

Tezozomoc, Hernando Alvarado, 1878. - Crónica mexicana precedida del Códice Ramirez, México.

-, 1943. - Crónica mexicana, Imprenta universitaria, México.

Torquemada, (Fray) Juan de, 1969. - Monarquía Indiana, 3 vol., Editorial Porrúa, México.

Vega Sosa, Constanza (coord.), 1979. - El recinto sagrado de México-Tenochtitlan. Excavaciones 1968-9 y 1975-6, SEP, INAH, México.

ZaNTwIJK, Rudolf van, 1965. - «Introducción al estudio de la división en quince partes de la sociedad azteca y su significación en la estructura interna ", Journal de la Société des Américanistes, 54 (2), pp. 211-222.

—, 1966. — « Los seis barrios sirvientes de Huitzilopochtli », Estudios de Cultura Nahuatl, 6, pp. 177-185.

—, 1985. - The Aztec Arrangement. The Social History of Pre-Hispanic Mexico, University of Oklahoma Press, Norman. 